Research Group: Macroeconomics

19 février 2009

\title{
Désinflation et chômage dans la zone Euro: Une analyse à l'aide d'un modèle VAR structurel
}

\section{PATRICK FÈVE, JULIEN MATHERON Et JeAN-GUILLAUME SAHUC}




\title{
Désinflation et chômage dans la zone euro : une analyse à l'aide d'un modèle VAR structurel*
}

\author{
Patrick FEVE ${ }^{\dagger}$, Julien MATHERON ${ }^{\ddagger}$, Jean-Guillaume SAHUC ${ }^{\S}$
}

19 février 2009

\begin{abstract}
Résumé
Cet article étudie les effets dynamiques des chocs de désinflation sur un ensemble de variables réelles et nominales de la zone Euro. A l'aide d'un modèle VAR structurel, nous identifions le choc de désinflation comme étant le seul choc ayant un effet permanent sur l'inflation à long terme ainsi que sur les variables nominales qui cointégrent avec elle. Nous montrons que ce choc a des effets récessionnistes importants, notamment sur l'investissement, et provoque une hausse persistante du taux de chômage et du taux d'intérêt réel. Nous complétons cette analyse en calculant des mesures d'inefficience sur les marchés des biens et du travail et nous montrons que ce sont principalement ces dernières qui ont prévalues. Ces conclusions sont robustes à différentes mesures et schémas d'identification des chocs de désinflation.
\end{abstract}

Mots-Clés : modèle VAR Structurel, restriction de long terme, désinflation

Classification JEL : C32, E31, E52

\footnotetext{
${ }^{*}$ Nous tenons à remercier deux rapporteurs anonymes et le rédacteur en chef (S. Roux) ainsi que F. Collard, M. Dupaigne, C. Pfister et F. Portier pour leurs précieuses remarques et suggestions. Nous restons seuls responsables des erreurs et imprécisions rémanentes. Les vues exprimées dans cet article sont nôtres et n'engagent en aucune façon la Banque de France.

${ }^{\dagger}$ Toulouse School of Economics (GREMAQ, IDEI et IUF) et Banque de France.

${ }^{\ddagger}$ Banque de France (DEMS-SEPS) et SDFi - Université de Paris-Dauphine.

${ }^{\S}$ Banque de France (DEMS-SEPS) et Audencia Ecole de Management.
} 


\section{Introduction}

Le taux d'inflation dans la zone Euro a fortement baissé durant les dernières décennies, passant de 12\% au début des années 1980 à $4 \%$ au début des années 1990. En dépit de certaines disparités, les inflations des différents pays de la zone présentent toutes une tendance marquée à la baisse sur cette période ${ }^{1}$. Durant cette phase de désinflation, le taux de chômage a augmenté de façon marquée et l'activité économique, notamment l'investissement, a sensiblement chuté dans la zone Euro. La figure 1 illustre ce fait pour la période 1980(1)-1990(1). Bien que l'évolution des variables réelles et nominales au cours de cette période puisse résulter d'autres facteurs et/ou chocs, un changement permanent dans la politique monétaire visant à réduire le taux d'inflation semble être un candidat explicatif naturel ${ }^{2}$. L'objectif de cet article est précisément d'évaluer l'importance quantitative de cette thèse.

Le lien entre activité réelle et inflation a fait l'objet d'une littérature empirique abondante. Une première approche utilise des régressions en coupe pour étudier les effets de l'inflation sur la croissance (voir par exemple Andres et Hernando, 1999, Kormendi et Meguire, 1985, McCandless et WEBER, 1995). Cette approche se concentre exclusivement sur des relations de long terme et fait abstraction des dynamiques d'ajustement à court terme. Une seconde approche étudie ces dynamiques de court terme à l'aide de modèles VAR Structurels (VARS) récursifs (voir CHRISTIANO et alii., 1999, pour un panorama exhaustif). Ces modèles supposent en général que ces chocs sont transitoires et ne représentent que des déviations de court terme vis à vis d'un comportement régulier de la politique monétaire. Ainsi, les modifications permanentes de l'inflation, telles qu'observées dans la zone Euro durant les trente dernières années (et surtout durant les années quatre-vingts), ne peuvent pas être appréhendées et analysées à l'aide de ce cadre empirique.

L'objectif de cet article se situe au carrefour de ces deux approches empiriques. Plus particulièrement, nous cherchons à étudier les réponses de court et moyen termes de l'économie faisant suite à des modifications permanentes de la cible d'inflation. La difficulté principale réside alors dans l'identification du choc de désinflation. A cette fin, nous abandonnons la représentation récursive discutée par CHRISTIANo et alii. [1999] et suivons la méthodologie VARS introduite par BULLARD et Keating [1995] ${ }^{3}$. Cette approche adapte au cas des chocs de désinflation la technique d'identification des chocs d'offre proposée par BlANCHARD et QUAH [1989]. Plus précisément, le choc de désinflation est identifié comme le seul choc ayant un effet permanent sur le taux d'inflation ainsi que sur les variables nominales qui cointègrent avec elle (inflation salariale, taux d'intérêt nominaux, taux de croissance de la monnaie dans notre cas). Notre spécification de référence impose de plus la neutralité de long terme du choc de désinflation, interprété ici comme un choc permanent de politique monétaire, suivant ainsi une version faible de la doctrine monétariste (l'inflation est toujours

1. Voir Blanchard et Muet [1993] et Cling et Meunier [1986] pour des analyses sur données françaises.

2. Fitoussi et Passet [2000] montrent que la politique monétaire a eu une grande influence sur l'évolution du chômage.

3. Voir aussi Andres et alii. [2002], Coenen et Vega [2001], Dolado et alii. [2000] et VlaAr [2005]. 
Figure 1 - Désinflation des années quatre-vingt
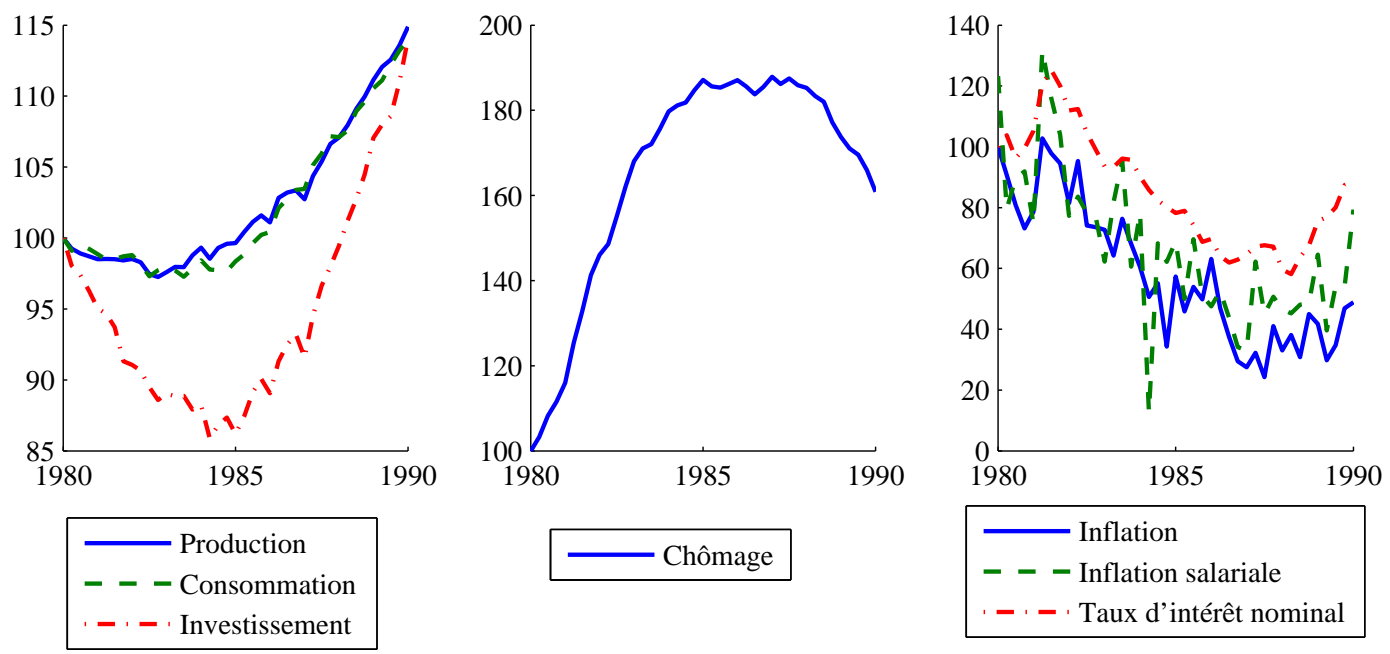

Notes : toutes les variables sont normalisées à 100 en $1980: 1$.

un phénomène monétaire dans le long terme). Une originalité de notre démarche est de combiner l'approche VARS avec le calcul de différentes mesures d'inefficience sur les marchés des biens et du travail basées sur un exercice de comptabilité du cycle à la GaLí et alii. [2003, 2007]. Cette analyse nous permet d'évaluer les conséquences d'un choc de désinflation et de mesurer quelles inefficiences ont joué un rôle dans la propagation de ses effets.

Comme l'a suggéré BlAnCHARD [2003], deux mécanismes concourent à expliquer les effets récessionnistes d'un choc de désinflation. En premier lieu, un tel choc peut entraîner un accroissement persistant du taux d'intérêt réel, se traduisant par une baisse de l'investissement. En second lieu, si les salaires nominaux sont plus visqueux que les prix, en raison de rigidités nominales ou réelles, un choc de désinflation peut entraîner une hausse du salaire réel et un accroissement du chômage. Une contribution du modèle VARS envisagé ici est d'intégrer différentes variables permettant d'étudier ces mécanismes de propagation. Plus précisément, nous prêtons une attention particulière au taux de chômage, au salaire réel, à l'investissement et au taux d'intérêt réel.

A partir de notre schéma d'identification, nous trouvons que le choc de désinflation a un effet persistant et important sur l'activité réelle. Il induit une baisse persistante du produit réel par tête, se traduisant par un ratio de sacrifice d'environ $5 \%$ à l'horizon de cinq ans, où ce nombre est mesuré traditionnellement comme la baisse cumulée de la production rapportée à la variation de l'inflation annualisée. En d'autres termes, la politique de désinflation aurait engendré un manque à gagner correspondant à $5 \%$ de la production potentielle en l'absence de cette politique. La réponse de l'investissement présente une baisse encore plus marquée, reflétant la réponse positive et persistante du taux d'intérêt réel (à court et à long termes). Enfin, la réponse dynamique du chômage suggère un arbitrage persistant (au moins dans le moyen terme) avec l'inflation, puisque le taux de chômage augmente de façon persistante pour atteindre un pic positif après deux ans. En revanche, le salaire réel présente une réponse positive dans le très court terme mais décrôit rapidement et s'ajuste à la 
baisse. Nous complétons ces résultats à l'aide du calcul de la réponse des marges sur les prix et les salaires. Cette dernière augmente de façon persistante et présente un profil en cloche, détériorant ainsi l'efficacité globale de l'économie. Nous évaluons finalement la robustesse de nos résultats à des spécifications alternatives du modèle VARS.

L'article est organisé comme suit. Dans une première section, nous présentons l'identification d'un choc de désinflation à partir d'un modèle VAR Structurel et nous discutons des résultats pour une représentation de référence. Une seconde section reporte différentes analyses de robustesse (prise en compte des chocs pétroliers, définitions alternatives de l'inflation, relâchement de certaines contraintes identifiantes). Une dernière section conclut.

\section{Caractérisation des chocs de désinflation dans la zone euro}

Dans cette section, nous présentons dans un premier temps la procédure d'identification du choc de désinflation à l'aide d'un modèle VARS. Nous exposons ensuite les effets dynamiques de ce choc sur un ensemble de variables d'intérêt. Enfin, nous menons un exercice de comptabilité du cycle à l'aide du modèle VARS et de restrictions théoriques additionnelles afin de caractériser les conséquences d'un choc de désinflation sur les marges de prix et de salaires.

\subsection{Identification à partir d'un modèle VAR structurel}

Nous étudions des données trimestrielles relatives à la zone euro, sur la période 1980(1)-2004(4). Nous nous intéressons au vecteur $Z_{t}$ défini comme

$$
Z_{t}=\left(\Delta \pi_{t}, \hat{y}_{t}, c_{t}-y_{t}, x_{t}-y_{t}, \hat{u}_{t}, \hat{w}_{t}, i_{t}^{l}-\pi_{t}, i_{t}^{l}-i_{t}^{s}, \gamma_{t}-\pi_{t}\right)^{\prime}
$$

où $\Delta \pi_{t}$ est la différence première du taux d'inflation $\pi_{t}, \hat{y}_{t}$ est la composante cyclique du logarithme $\mathrm{du}$ PIB,$c_{t}-y_{t}$ est le logarithme du rapport consommation sur PIB, $x_{t}-y_{t}$ est le logarithme $\mathrm{du}$ rapport investissement sur PIB, $\hat{u}_{t}$ est la composante cyclique du taux de chômage, $\hat{w}_{t}$ est la composante cyclique du logarithme du salaire réel, $i_{t}^{l}$ est le taux d'intérêt nominal de long terme, $i_{t}^{s}$ est le taux d'intérêt nominal de court terme et $\gamma_{t}$ est le taux de croissance de M3 ${ }^{4}$. Ce dernier est corrigé de la réunification allemande $(1990(3))$ et de l'entrée de la Grèce dans la zone Euro (2001(1)). Plus précisément, $\gamma_{t}$ est obtenu comme le résidu de la régression de la série d'origine sur des variables indicatrices correspondant à ces deux dates.

Le choix des variables dans $Z_{t}$ est en partie inspiré de Blanchard [2003]. Ce dernier identifie deux conséquences possibles d'une désinflation : en premier lieu, un accroissement du taux d'intérêt réel, se traduisant par une hausse du prix du capital et une baisse de l'investissement; en second lieu, un accroissement des salaires réels, dû à des rigidités nominales ou réelles, et se traduisant

4. Voir l'annexe A pour une définition détaillée des données et de leurs sources. Les données brutes sont reportées sur le graphique A.1. 
Tableau 1. Tests de racine unitaire sur les variables réelles

\begin{tabular}{lcc}
\hline \hline & ADF & PP \\
\cline { 2 - 3 }$u_{t}$ & -2.61 & -3.15 \\
& {$[9.43]$} & {$[2.63]$} \\
$y_{t}$ & -2.78 & -3.26 \\
& {$[20.88]$} & {$[7.91]$} \\
$c_{t}-y_{t}$ & -2.76 & -2.76 \\
& {$[6.70]$} & {$[6.70]$} \\
$i_{t}-y_{t}$ & -3.00 & -4.07 \\
& {$[3.85]$} & {$[0.17]$} \\
$w_{t}$ & -0.72 & -0.72 \\
& {$[96.87]$} & {$[96.87]$} \\
\hline
\end{tabular}

\begin{abstract}
Notes : ADF : test de Dickey-Fuller Augmenté. Les retards sont selectionnés à l'aide du critère Hannan-Quinn. PP : tests de Phillips-Perron, avec spécification AR spectrale estimée par MCO, où les retards sont là aussi séléctionnés à l'aide du critère Hannan-Quinn. Pour $u, c-y$ et $i-y$, les tests autorisent seulement une constante tandis que pour $y$ et $w$ ils autorisent à la fois une constante et une tendance linéaire. Dans tous les cas, l'hypothèse nulle est la racine unitaire. Les seuils des statistiques du test sont entre crochets et en pourcentage.
\end{abstract}

par une hausse du chômage. Les variables incluses dans $Z_{t}$ permettent en principe de capter ces mécanismes. Par ailleurs, le taux court est inclus dans l'analyse pour deux raisons. En premier lieu, nous voulons étudier la dynamique du taux court, vu comme instrument de la politique monétaire (en complément de M3). En second lieu, en considérant à la fois le taux court et le taux long, nous pouvons quantifier le niveau de crédibilité de la politique de désinflation. En particulier, une réaction du taux long plus prononcée que celle du taux court peut être interprétée comme un signe de crédibilité.

Les composantes cycliques du PIB, du salaire réel et du chômage sont obtenues à l'aide du filtre à bande passante de Christiano et FitzGerald [2003], en éliminant les mouvements de période supérieure à 60 trimestres. Ce faisant, nous suivons la définition du moyen terme adoptée par BLANChard [1997] et par Staiger et alii. [2002 ${ }^{5}$. Un intérêt du filtre de Christiano et Fitzgerald [2003] est qu'il permet d'éliminer la présence d'une racine unitaire dans la série originale. De fait, si les tests usuels de racine unitaire rejettent sans ambiguïté l'hypothèse de racine unitaire pour les ratios consommation-produit et investissement-produit, ils donnent des résultats plus ambigus pour le produit, le taux de chômage et le salaire réel, comme le suggèrent les résultats du tableau 1. Pour compléter la discussion de la spécification de $Z_{t}$, il est aussi important d'évaluer si l'inflation et les autres variables nominales incluses dans le vecteur $Z_{t}$ peuvent être caractérisées par un processus intégré d'ordre un dans notre échantillon. A cette fin, nous effectuons les mêmes tests de racine unitaire que ci-dessus. Les résultats sont reportés dans le tableau 2.

5. Galí et alii. [2003] adoptent la même procédure d'extraction de la composante cyclique. 
Tableau 2. Tests de racine unitaire sur les variables nominales

\begin{tabular}{lccccc}
\hline \hline & \multicolumn{3}{c}{ Niveau } & & \multicolumn{2}{c}{ Différence première } \\
\cline { 2 - 3 } \cline { 5 - 6 }$\pi_{t}$ & ADF & PP & & ADF & PP \\
\cline { 2 - 3 }$i_{t}^{l}$ & -1.99 & -2.67 & & -9.66 & -14.23 \\
& {$[29.09]$} & {$[8.30]$} & & {$[0.00]$} & {$[0.00]$} \\
$i_{t}^{s}$ & -0.78 & -0.46 & & -5.34 & -9.67 \\
& {$[81.93]$} & {$[89.34]$} & & {$[0.00]$} & {$[0.00]$} \\
$\gamma_{t}$ & -1.19 & -1.00 & & -6.17 & -6.17 \\
& {$[67.70]$} & {$[74.32]$} & & {$[0.00]$} & {$[0.00]$} \\
& -2.27 & -6.11 & & -7.83 & -27.84 \\
$i_{t}^{l}-\pi_{t}$ & {$[18.44]$} & {$[0.00]$} & & {$[0.00]$} & {$[0.00]$} \\
& & & & \\
$i_{t}^{l}-i_{t}^{s}$ & -3.30 & -4.26 & & & \\
& {$[1.74]$} & {$[0.09]$} & & & \\
$\gamma_{t}-\pi_{t}$ & -3.22 & -3.07 & & & \\
& {$[2.19]$} & {$[3.13]$} & & & \\
\hline
\end{tabular}

Notes : ADF : test de Dickey-Fuller Augmenté. Les retards sont selectionnés à l'aide du critère Hannan-Quinn. PP : tests de Phillips-Perron, avec spécification AR spectrale estimée par MCO, où les retards sont là aussi séléctionnés à l'aide du critère Hannan-Quinn. Nous incluons une constante, mais pas de tendance déterministe. Dans tous les cas, l'hypothèse nulle est la racine unitaire. Les seuils des statistiques du test sont entre crochets et en pourcentage.

De manière générale, les tests suggèrent que les différentes variables nominales incluses dans $Z_{t}$, c'est à dire l'inflation, les taux d'intérêt nominaux de court et long termes et le taux de croissance de la monnaie, présentent toutes une racine unitaire. Nous obtenons de plus que ces variables en différence première sont toutes stationnaires. A partir de ces propriétés univariées, nous étudions dans un second temps s'il existe une relation de long terme entre ces variables. Nous testons alors si le taux d'intérêt réel de long terme ex-post $\left(i_{t}^{l}-\pi_{t}\right)$, l'écart taux long-taux court $\left(i_{t}^{l}-i_{t}^{s}\right)$ et le taux de croissance des encaisses réelles $\left(\gamma_{t}-\pi_{t}\right)$ sont stationnaires. De façon générale, les résultats sont plutôt favorables à la stationarité de ces variables, ce qui suggère qu'elles partagent la même tendance stochastique de long terme. Nous imposons donc cette relation de cointégration dans le modèle VAR Structurel.

Nous supposons que le vecteur $Z_{t}$ évolue selon le processus VAR

$$
Z_{t}=A_{0}+\sum_{i=1}^{\ell} A_{i} Z_{t-i}+e_{t}
$$

où $\ell$ est le nombre de retards et $e_{t}$ est un bruit blanc de matrice de variance-covariance $\Sigma$. Le nombre de retards $\ell$ est sélectionné à l'aide de critères d'information standards. Dans nos différentes 
expériences, nous obtenons $\ell=1$ ou $\ell=2$.

Notre hypothèse d'identification des chocs de désinflation consiste à imposer que seuls ces derniers peuvent affecter le niveau de l'inflation à long terme ${ }^{6}$. Cette restriction d'identification est analogue à celle initialement proposée par BLANCHARD et QUAH [1989] dans le cadre des chocs d'offre. Formellement, nous supposons que les innovations canoniques $e_{t}$ sont une combinaison linéaire des chocs structurels $\eta_{t}$, soit $e_{t}=S \eta_{t}$, où $S$ est une matrice de passage. Dans un premier temps, nous supposons que les chocs structurels sont orthogonaux et normalisons ces derniers de telle sorte que $\mathrm{E}\left\{\eta_{t} \eta_{t}^{\prime}\right\}=I_{m}$, où $I_{m}$ est la matrice identité et $m$ la dimension de $Z_{t}$. Posons alors $C(L)=B(L) S$, où

$$
B(L)=\left(I_{m}-A_{1} L-\cdots-A_{\ell} L^{\ell}\right)^{-1} .
$$

Dans un second temps, étant donné l'ordre des variables dans $Z_{t}$ dans l'équation (1), nous imposons que seul le premier élément de la première ligne de $C(1)$ soit a priori non nul. En pratique, nous sélectionnons $S$ de telle sorte que $C(1)$ soit la matrice triangulaire inférieure obtenue par la décomposition de Cholesky de $B(1) \Sigma B(1)^{\prime}$. Les chocs de désinflation ainsi identifiés sont invariants à toute transformation orthonormale de la matrice extraite de $S$ en éliminant la première ligne et la première colonne de cette dernière.

Compte tenu de la spécification retenue, le choc de désinflation aura un effet permanent non seulement sur l'inflation mais aussi sur les autres variables nominales incluses dans $Z_{t}$ qui cointègrent avec elle, c'est-à-dire les taux d'intérêt nominaux de court et long termes et le taux de croissance de M3. De plus, en spécifiant la composante cyclique du produit, du salaire réel et du taux de chômage dans $Z_{t}$ et en considérant les rapports consommation-produit et investissement-produit, nous imposons implicitement que les chocs de désinflation ne peuvent pas avoir d'effets à long terme sur les variables réelles du modèle VAR Structurel. En d'autres termes, les chocs de désinflation peuvent s'interpréter comme des chocs permanents sur la politique monétaire qui sont neutres dans le long terme.

\subsection{Les effets agrégés d'un choc de désinflation}

Avant de présenter les réponses des variables agrégées, nous étudions le comportement temporel du choc de désinflation. Suivant certaines études empiriques (STOCK et WATson, 2007, et Cogley et SARGEnt, 2007) ainsi que des spécifications plus structurelles (IRElAnd, 2007, SMETs et Wouters,

6. Il faut noter que le schéma d'identification adopté dans ce papier implique que le choc de désinflation peut avoir lieu à toutes les périodes de l'échantillon. Cette stratégie empirique diffère d'une approche narrative (voir Romer et Romer, 1989 et 1994) qui sélectionne un petit nombre d'épisodes associés à des politiques actives de désinflation. Comme l'ont noté Christiano et alii.. [1999], un avantage de l'approche narrative réside dans le fait que l'économètre n'a pas à spécifier explicitement une règle monétaire ou à imposer un schéma particulier d'identification afin d'identifier les réponses de l'économie. Une telle approche n'est pas possible dans notre cas dans la mesure où il n'existe pas d'analogues des minutes du FOMC ni pour les banques centrales nationales de la zone euro ni pour la BCE. 
2005, et De Walque, Smets et Wouters, 2006), nous supposons que la cible implicite d'inflation de la banque centrale évolue selon une marche aléatoire

$$
\pi_{t}^{\star}=\pi_{t-1}^{\star}+\sigma_{\pi} \eta_{t}^{\pi}
$$

où $\eta_{t}^{\pi}$ est le choc de désinflation identifié à partir du modèle (2) selon notre schéma d'identification et $\sigma_{\pi}$ est l'élément $1 \times 1$ de $S$. En procédant de la sorte, nous redimensionnons les chocs de désinflation pour les rendre de même "taille" que les données. La valeur initiale de $\pi_{t}^{\star}$ est fixée à la moyenne de l'inflation observée sur la période 1975(1)-1979(4). Cette représentation est évidemment une simplification, introduite à des fins illustratives. En effet, le modèle VARS nous permet d'identifier l'innovation structurelle de la cible implicite d'inflation. En revanche, le modèle VARS reste silencieux quant au comportement du banquier central et ne peut donc pas être utilisé pour identifier structurellement le processus suivi par cette cible implicite.

Il convient de remarquer que, pour l'essentiel de la période d'estimation considérée dans cette étude (1980(1)-2004(4)), les pays constituant la zone euro avaient chacun une banque centrale et ne mettaient pas en œuvre une politique monétaire unique. Le choix de modélisation retenu ici, d'ailleurs adopté dans la majeure partie de la littérature (en particulier dans la littérature SVAR, comme chez Peersman et Smets, 2001 ou DSGE, comme chez Smets et Wouters, 2003), est de traiter la banque centrale unique sous-jacente à l'équation (3) comme une banque centrale virtuelle, moyenne pondérée des 12 banques centrales nationales de l'époque. Cette approche a le défaut de gommer l'hétérogénéité des politiques monétaires précédant l'introduction de l'euro. Toutefois, cette approximation est cohérente avec la rapide adoption par de nombreux pays d'objectifs de politique monétaire convergents, en particulier, la réduction du niveau de l'inflation, comme le suggère le graphique A.2 qui reporte les trajectoires d'inflation des 12 pays membres avant l'adoption de l'euro. Ce graphique illustre clairement que les pays membres ont quasiment tous procédé à des politiques de désinflation en même temps ou, à tout le moins, sur la même période. Les épisodes de désinflation identifiés par BALL [1994] et ANDERSON et WAScher [1999] pour les pays membres les plus importants confirment cette observation. Par exemple, ces études identifient les épisodes 1981-1986 pour la France, 1980-1986 pour l'Allemagne et 1980-1987 pour l'Italie. En outre, vers la fin de l'échantillon, du fait des critères de convergence imposés par le traité de Maastricht, les taux d'inflation convergent quasi mécaniquement vers un niveau commun. De ce fait, il ne semble pas y avoir de changement de régime entre les périodes pre et post 1999. C'est en tous les cas l'hypothèse de travail retenue dans cet article.

La figure 2 reporte cette cible. Les aires grisées correspondent aux différents épisodes de récession, obtenus en utilisant une méthode similaire à celle de WATSON $[1994]^{7}$. Ces épisodes de récession sont 1980(1)-1981(1), 1982(1)-1982(4), 1992(1)-1993(2), et 2002(3)-2003(2). Ces dates coïncident à peu près avec celles identifiées par le "Euro Area Business Cycle Dating Committee". La seule

7. Plus précisément, les dates de récession sont identifiées en utilisant l'algorithme de Bry-Boschan sur le logarithme du niveau du PIB réel par tête. 
FiguRE 2 - Cible d'inflation identifiée par le modèle VARS

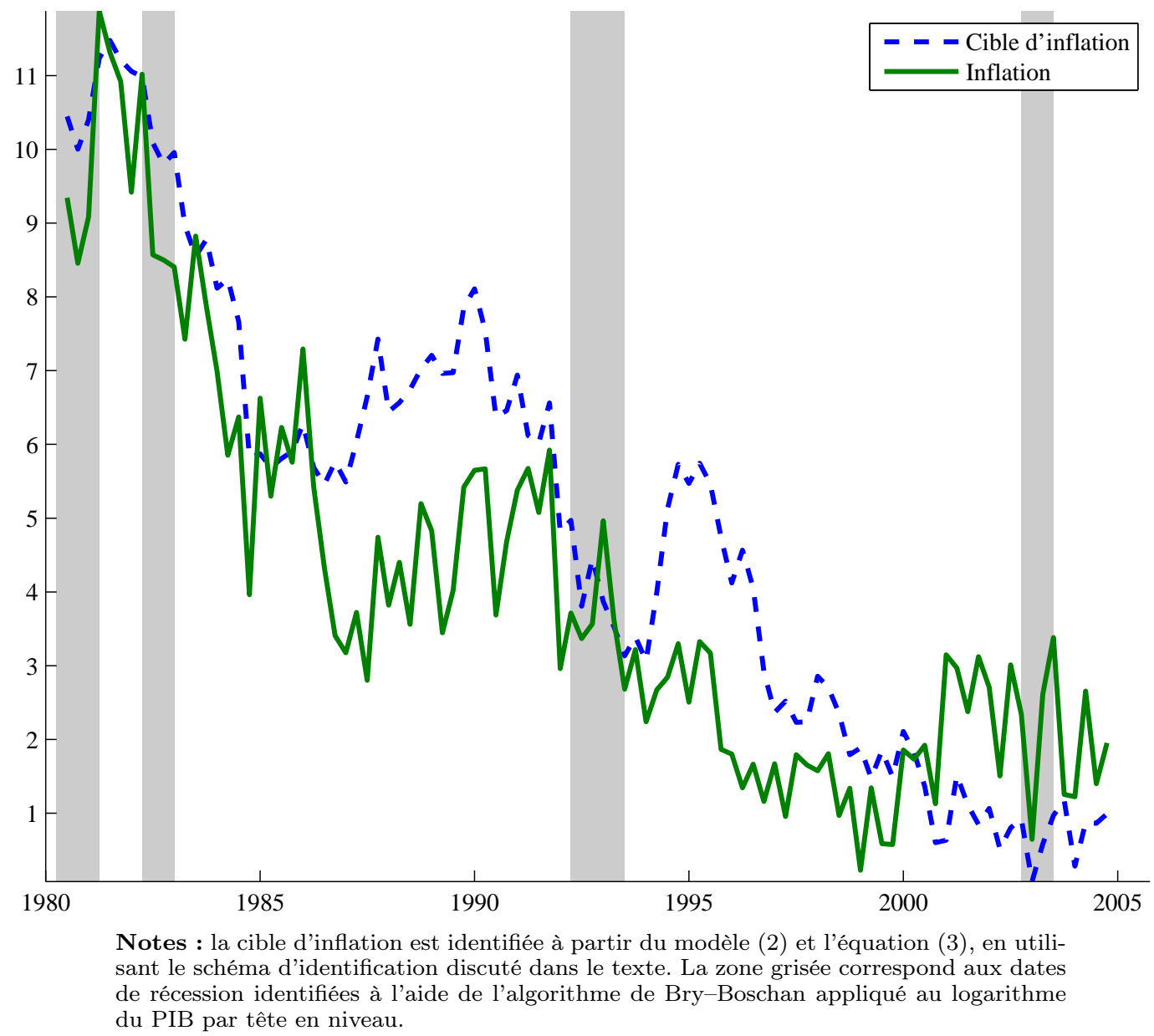

exception est la récession du début des années quatre-vingt qui est séparée en deux récessions avec notre procédure. La différence provient du fait que nous utilisons un algorithme mécanique de sélection à partir du PIB par tête, alors que le comité adopte une procédure de jugement.

Cette figure met en évidence la grande similitude d'évolution du taux d'inflation de la zone euro et de la cible implicite d'inflation poursuivie par la politique monétaire. Il faut rappeler que notre schéma d'identification suppose une origine monétaire de la composante non-stationnaire de l'inflation. Ces deux variables tendent toutes les deux à baisser fortement dans les premières moitiés des années quatre-vingts et quatre-vingt-dix. La politique de désinflation ainsi identifiée par le modèle VARS peut être associée à deux grandes récessions de la zone Euro, c'est à dire celles de 1982(1)-1982(4) et de 1992(1)-1993(2).

Dans l'esprit d'IRELAND [2007], nous complétons cette analyse en simulant le modèle VAR sous l'hypothèse contre-factuelle que les chocs de désinflation ont été identiquement nuls sur l'échantillon. Nous reconstruisons alors les trajectoires des variables d'intérêt. Les résultats sont reportés sur la figure 3 pour les composantes cycliques du produit, de la consommation, de l'investissement et du chômage ainsi que pour l'inflation, le taux de croissance de la monnaie et les taux d'intérêt nominaux 
Figure 3 - Trajectoires des variables avec ou sans chocs de désinflation

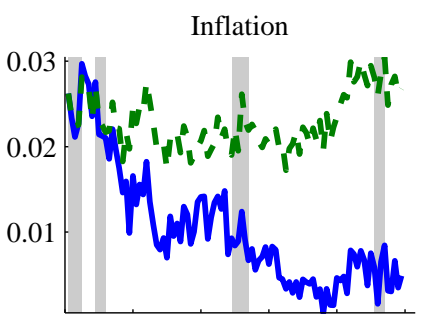

198019851990199520002005

Investissement

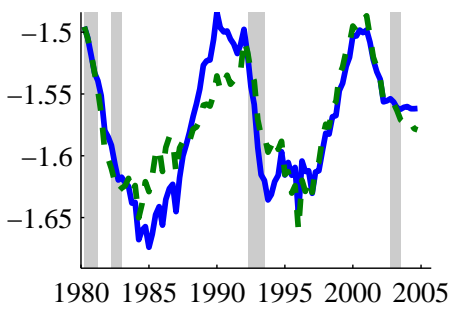

Taux de chômage

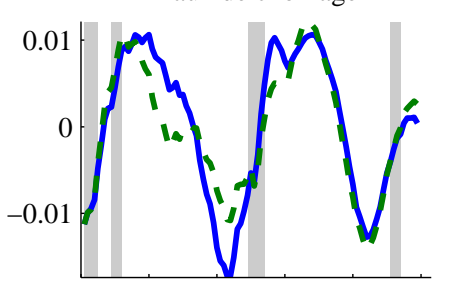

198019851990199520002005
Production

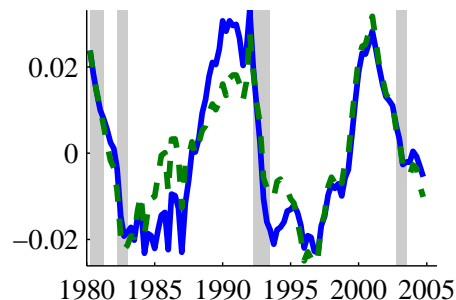

Taux d'intérêt nominal long

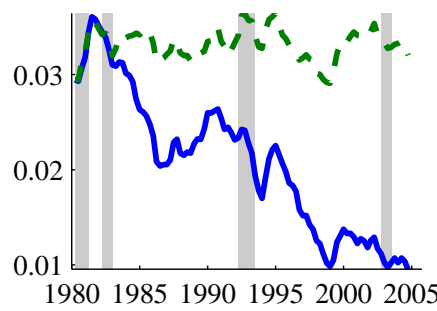

Croissance de la monnaie (M3)

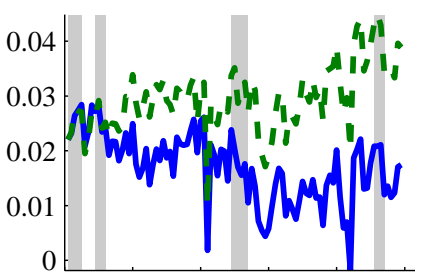

198019851990199520002005
Consommation

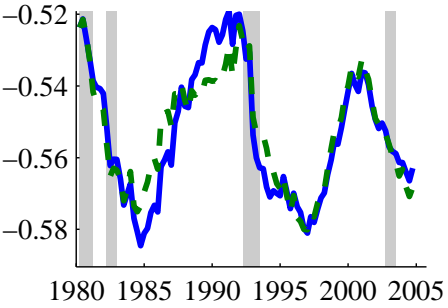

Taux d'intérêt nominal court

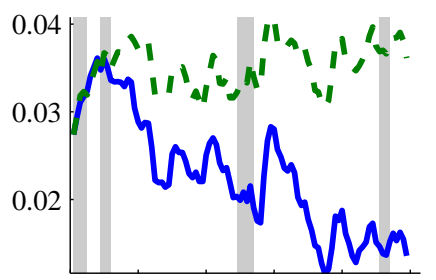

198019851990199520002005

Salaire réel

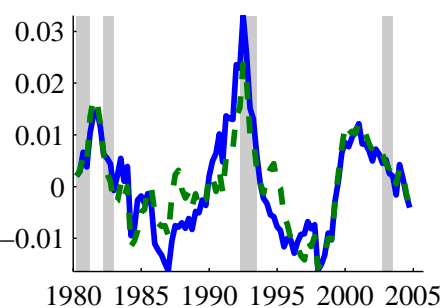

Notes : les lignes continues correspondent au cas avec choc de désinflation; les lignes brisées correspondent au cas où le choc de désinflation est identiquement nul sur l'échantillon.

de court et de long termes. Le graphique illustre que sur la période 1982-1988, les composantes cycliques du produit, de la consommation et de l'investissement auraient été plus élevées sans chocs de désinflation, en restant toutefois sous leur niveau tendanciel. Concernant le chômage, l'effet de la désinflation se fait essentiellement sentir dans la première moitié des années 1980. Ces différences semblent moins marquées sur la fin de l'échantillon, après 1995. En ce qui concerne les variables nominales, la différence est, par construction, beaucoup plus criante. Il est cependant intéressant de remarquer qu'en l'absence de chocs de désinflation, l'inflation et le taux de croissance de la monnaie se seraient fortement accrus passé 2000. Les chocs de désinflation ont servi à contrebalancer les effets inflationnistes des autres chocs.

Pour mieux comprendre les résultats précédents, nous présentons maintenant les fonctions de réponse à un choc de désinflation dans notre spécification (1) de $Z_{t}$. Pour chacune des réponses, nous calculons les intervalles de confiance à l'aide d'une technique de bootstrap ${ }^{8}$. Afin de simplifier l'in-

8. Nous calculons $N=1000$ simulations bootstrap du modèle VAR structurel en construisant $N$ nouvelles séries temporelles des résidus canoniques, notées $\left\{e_{t}(i)\right\}_{t=1}^{T}, i=1, \ldots, N$, où le $t^{\text {ème }}$ élément de $\left\{e_{t}(i)\right\}_{t=1}^{T}$ est tiré avec remise dans $\left\{e_{t}\right\}_{t=1}^{T}$. En utilisant les paramètres estimés du modèle VAR et les valeurs historiques observées comme valeurs initiales, nous construisons alors $N$ séries temporelles de $Z_{t},\left\{Z_{t}(i)\right\}_{t=1}^{T}$. Pour chaque simulation, le modèle 
terprétation, nous reportons les réponses de l'inflation, du taux de croissance de la monnaie et des taux d'intérêt nominaux ainsi que les réponses des composantes cycliques de la consommation, de l'investissement ${ }^{9}$. Ces réponses sont obtenues à partir de celles des différents éléments du vecteur $Z_{t}$. Ainsi, la réponse de l'inflation est obtenue en cumulant la réponse du premier élément de $Z_{t}$. De même, celle de la consommation s'obtient en additionnant la réponse de la deuxième et de la troisième variable de $Z_{t}$.

La figure 4 reporte les réponses dynamiques des neuf variables incluses dans $Z_{t}$ à un choc permanent de désinflation. Elles sont toutes normalisées de sorte que l'inflation trimestrielle est réduite de $1 \%$ à long terme. Nous considérons dans un premier temps les réponses des variables nominales. L'inflation et le taux de croissance de la monnaie présentent des profils très similaires. Toutes les deux commencent par diminuer fortement, puis fluctuent avant de converger lentement vers leur nouvelle valeur de long terme. Le fait que l'inflation et le taux de croissance de la monnaie présentent dans le très court terme des réponses aussi proches conforte l'idée que les chocs de désinflation peuvent être interprétés comme des chocs permanents sur la politique monétaire. Dans le même temps, le taux d'intérêt nominal de court terme ne répond pratiquement pas à l'impact, puis décroît régulièrement et sur-ajuste légèrement sa nouvelle valeur de long terme. De manière contrastée, le taux nominal de long terme baisse significativement à l'impact, ce qui implique que l'écart de taux diminue significativement à court terme. Ce résultat suggère que les agents ont en partie intégré la baisse de l'inflation engendrée par la politique de désinflation.

Concernant les variables réelles, nous obtenons les résultats suivants. Tout d'abord, le choc de désinflation a un effet négatif, persistant et significatif sur le produit par tête. Plus précisément, une baisse de un pour cent de l'inflation à long terme induit une baisse de plus de deux pour cent du produit après deux ans. Au bout de cinq ans, la réponse du produit s'annule. Le ratio de sacrifice associé est important. Ce dernier est défini de façon usuelle dans la littérature comme la réponse cumulée du produit divisée par la baisse de l'inflation annuelle (ici 4\%). Après cinq ans, le ratio de sacrifice estimé est égal à $4.9 \%$ du produit cumulée avec un écart-type de 2.29. Le ratio de sacrifice augmente régulièrement avec l'horizon de la réponse pour se stabiliser approximativement douze ans après le choc de désinflation. Il faut noter que le produit et l'inflation varient dans le même sens, ce qui permet d'interpréter le choc de désinflation comme un choc de demande. La consommation baisse suite au choc, mais de façon un peu moins marquée que le produit, reflétant ainsi une phénomène de lissage. De façon contrastée, l'investissement présente une baisse deux fois plus forte que celle du produit.

Afin de mieux comprendre cette baisse de l'investissement, il est instructif de regarder les réponses des taux d'intérêt réels ex-ante (à court et à long termes). Celles-ci sont reportées dans la figure

\footnotetext{
VAR spécifié dans l'équation (2) est estimé et les fonctions de réponse sont calculées à partir de la matrice de passage estimée à chaque simulation.

9. Notre spécification de $Z_{t}$ implique que la consommation, l'investissement et le produit partagent la même composante permanente.
} 
Figure 4 - Réponses dynamiques à un choc de désinflation

Inflation
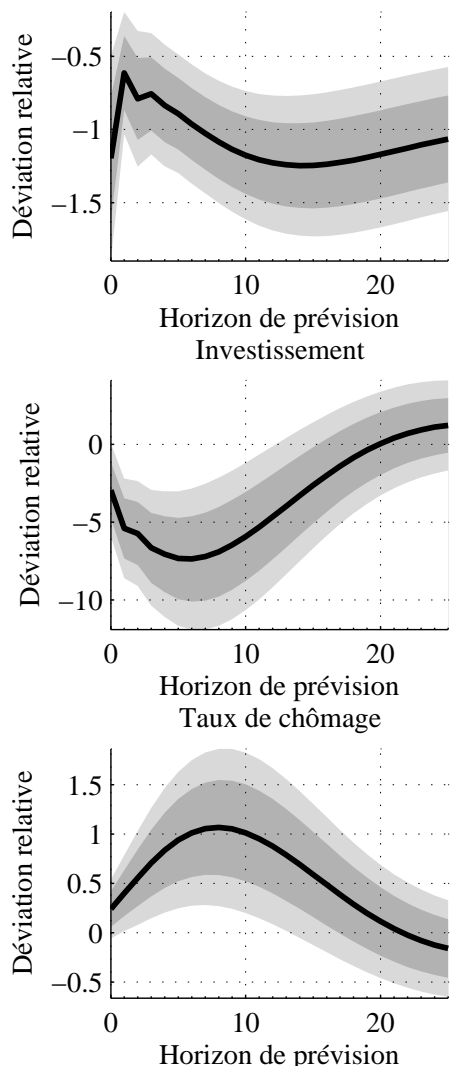

Production

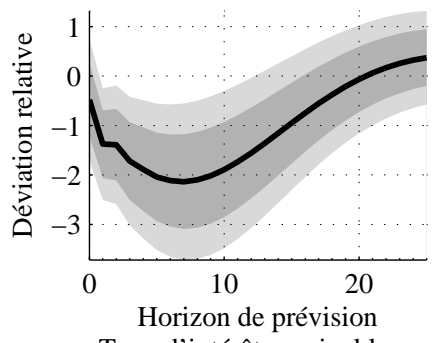

Taux d'intérêt nominal long
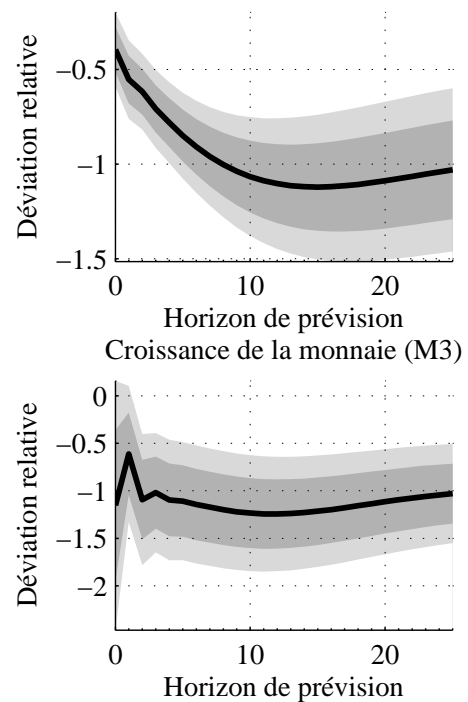

Consommation

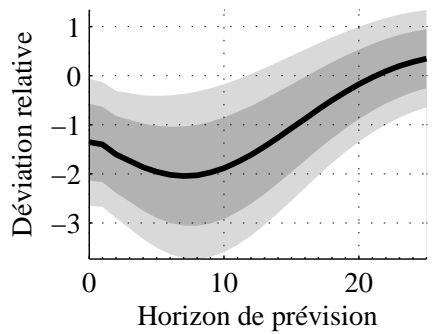

Taux d'intérêt nominal court

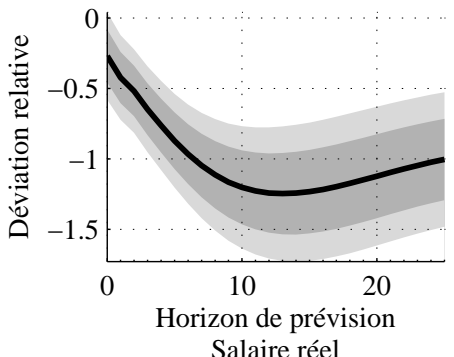

Salaire réel

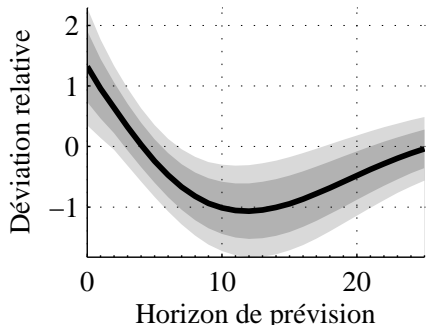

Notes : les aires grisées correspondent à l'intervalle de confiance à $90 \%$ (clair) et à $68 \%$ (foncé), obtenus à l'aide d'une technique de Bootstrap. Afin de faciliter l'interprétation, la taille du choc de désinflation est normalisée de sorte que la réponse asymptotique de l'inflation soit égale à $-1 \%$.

5. Pour les deux cas, les taux ex-ante sont obtenus comme la différence entre le taux nominal et l'inflation anticipée à un pas, cette dernière étant calculée à l'aide de la réponse avancée de l'inflation dans le modèle VARS. Les deux taux réels répondent positivement, leur réponse à l'impact étant approximativement égale à la moitié (en valeur absolue) de la réponse de long terme de l'inflation. De plus, ces réponses positives apparaissent persistantes. Ceci suggère que les politiques de désinflation ont augmenté de façon persistante le coût du capital, entraînant ainsi une baisse importante de l'investissement. Il convient toutefois de remarquer que ces réponses ne sont pas précisément estimées.

Le taux de chômage ne réagit pas à l'impact mais présente dans les périodes suivantes un profil en cloche. Cette réponse du taux de chômage suggère un coût réel important de la politique de désinflation, puisque la composante cyclique du chômage s'accroît d'un point après deux ans. Cette dynamique suggère l'existence d'un arbitrage inflation-chômage persistant. Ce point est mis en évidence dans la figure 6 qui reporte la dynamique d'ajustement impliquée par le modèle VARS autour de la courbe de Phillips de long terme.

Dans un premier temps, l'inflation baisse tandis que le chômage augmente. Au cours de cet épisode, 
Figure 5 - Réponses dynamiques des taux d'intérêt réels à un choc de désinflation
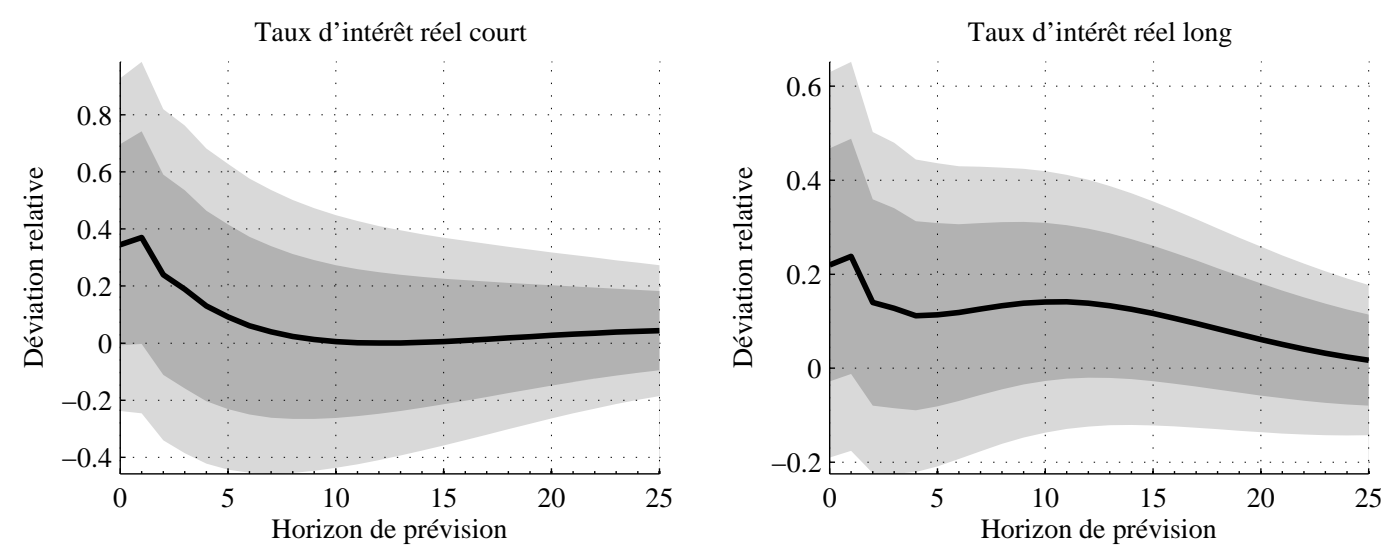

Notes : les aires grisées correspondent à l'intervalle de confiance à $90 \%$ (clair) et à $68 \%$ (foncé), obtenus à l'aide d'une technique de Bootstrap. Afin de faciliter l'interprétation, la taille du choc de désinflation est normalisée de sorte que la réponse asymptotique de l'inflation soit égale à $-1 \%$.

en effet, les salaires réels augmentent instantanément (voir figure 4). Après quelques périodes, le taux de chômage revient à sa valeur de long terme tandis que l'inflation rejoint sa nouvelle cible. Le retour du chômage à son niveau initial est permis par la baisse du salaire réel qui joue ici le rôle de la force de rappel. Ce graphique illustre bien que même s'il y a neutralité à long terme de la politique de désinflation, la dynamique d'ajustement du chômage est extrêmement lente.

Comme l'a suggéré BLANCHARD [2003], les effets négatifs de la politique de désinflation sur l'emploi peuvent provenir de deux sources. Tout d'abord, la désinflation peut provoquer une hausse du salaire réel, lorsque les salaires nominaux présentent une forte inertie à court terme. Ensuite, en augmentant le coût du capital (le taux d'intérêt réel de long terme), une telle politique induit une baisse persistante de l'accumulation de capital physique. Dans la mesure où le travail et le capital sont des facteurs de production complémentaires, la baisse de l'investissement se traduit par une hausse du chômage. Ces deux effets sont présents dans le modèle VARS, puisque à la fois le salaire réel (voir la figure 4) et le taux d'intérêt réel (voir la figure 5) augmentent à court terme. Toutefois, seule la réponse du taux d'intérêt réel est persistante.

Nous complétons ces résultats en calculant la contribution du choc de désinflation à la variance des neuf variables incluses dans $Z_{t}$. Le tableau 3 reporte cette décomposition de variance pour différents horizons.

Concernant les variables nominales, le choc de désinflation est le choc dominant de leur fluctuations et ce, même dans le court terme (avec une exception pour le taux nominal de court terme). Par exemple, ce choc représente $69 \%$ de l'inflation, $67 \%$ du taux d'intérêt nominal de court terme, $86 \%$ du taux d'intérêt nominal de long terme et $41 \%$ du taux de croissance de la monnaie après trois ans. Au bout de cinq ans, les chocs de désinflation représentent entre $50 \%$ et $90 \%$ des fluctuations de ces variables nominales et par construction cette contribution augmente avec l'horizon. Bien que notre schéma d'identification impose la neutralité à long terme des chocs sur la politique de 
Figure 6 - Dynamique d'ajustement autour de la courbe de Phillips de long terme

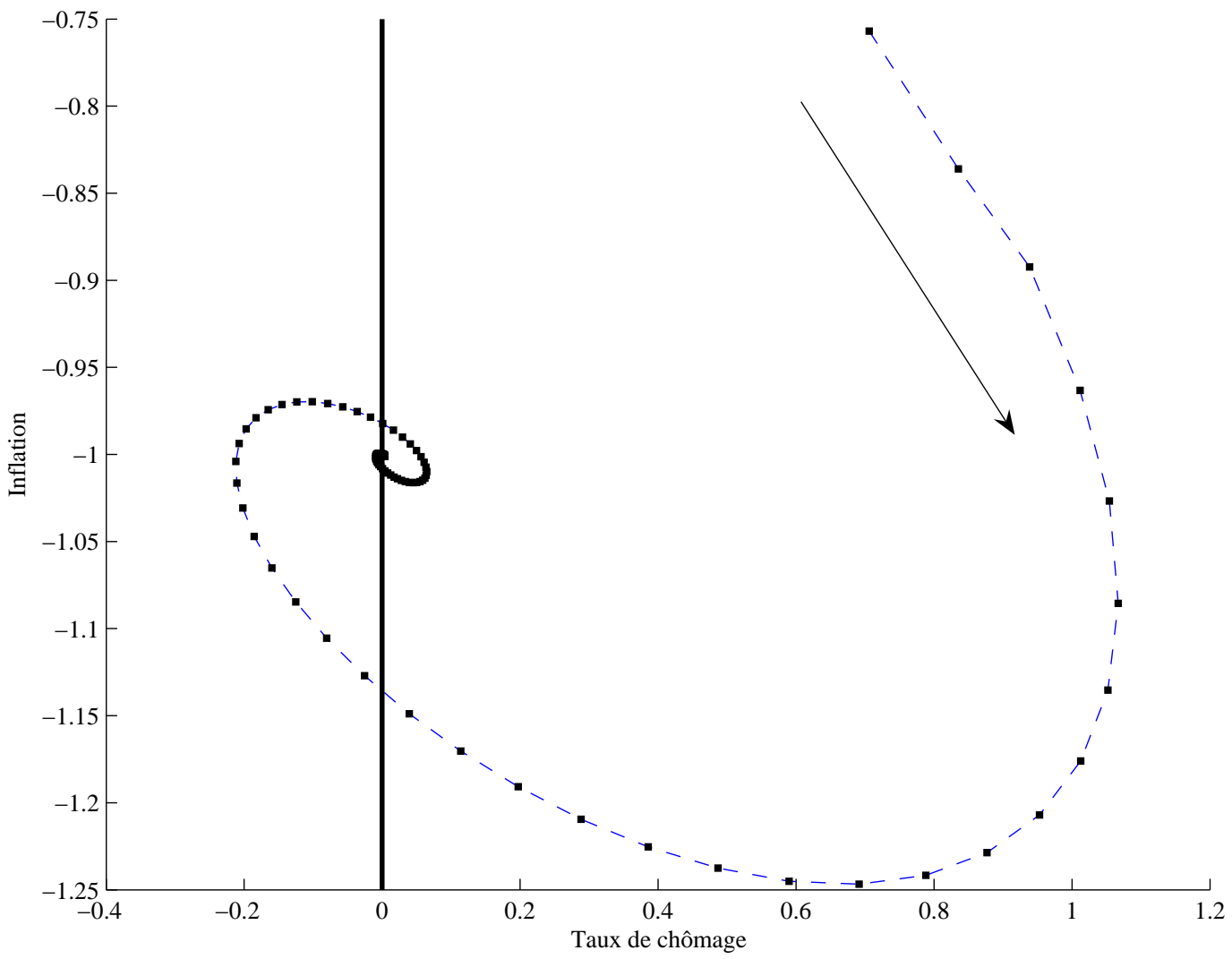

Notes : les réponses correspondant à la première année consécutive au choc de désinflation sont omises du graphique afin d'en faciliter la lecture. La flèche indique le sens d'enroulement de la courbe de Phillips de court terme.

désinflation, ceux-ci ont un effet non négligeable sur les variables réelles. Ils représentent $28 \%$ de la variance de la composante cyclique du produit et $38 \%$ de celle de l'investissement après trois ans. Cette contribution apparaît de même ampleur pour la consommation et le taux de chômage (entre $25 \%$ et $30 \%$ ) pour le même horizon. Pour des horizons plus longs, par exemple après dix ans, cette contribution se situe autour de $25 \%$ pour les variables réelles. Ce résultat reflète la réponse graduelle du secteur productif aux chocs sur la politique monétaire.

\subsection{Désinflation et mesures d'inefficience}

Les réponses obtenues à partir du modèle (2) peuvent être utilisées afin de mener un exercice de comptabilité du cycle et de fournir des mesures d'inefficience dans la zone euro. Les éléments permettant de telles mesures sont présents dans le modèle (2) puisque le vecteur $Z_{t}$ inclut différentes variables relatives à ces mesures d'inefficience. Malheureusement, le modèle VAR ne permet pas de dériver formellement et directement de telles mesures. Pour ce faire, nous devons coupler certaines restrictions issues de la théorie avec les réponses des différentes variables contenues dans $Z_{t}$. A cette 
Tableau 3. Décomposition de la variance

\begin{tabular}{cccccc}
\hline \hline & \multicolumn{5}{c}{ Horizon de prévision } \\
\cline { 2 - 6 } & 0 & 12 & 20 & 40 & $\infty$ \\
\hline$\pi_{t}$ & 36.37 & 68.76 & 78.68 & 85.69 & 100.00 \\
& {$[1.16,59.73]$} & {$[23.06,85.17]$} & {$[41.56,90.16]$} & {$[63.43,94.73]$} & {$[-]$} \\
$\hat{y}_{t}$ & 1.82 & 28.34 & 27.14 & 25.72 & 25.71 \\
& {$[0.03,23.30]$} & {$[1.85,58.59]$} & {$[2.35,58.80]$} & {$[2.65,58.29]$} & {$[2.64,58.28]$} \\
$\hat{c}_{t}$ & 13.47 & 26.38 & 25.59 & 25.20 & 25.19 \\
& {$[0.14,41.30]$} & {$[2.59,59.97]$} & {$[3.20,59.96]$} & {$[3.69,58.62]$} & {$[3.65,59.31]$} \\
$\hat{x}_{t}$ & 9.22 & 38.17 & 35.06 & 32.28 & 32.23 \\
& {$[0.05,30.16]$} & {$[3.44,61.63]$} & {$[3.95,61.00]$} & {$[4.30,60.16]$} & {$[4.31,60.17]$} \\
$i_{t}^{l}$ & 29.81 & 86.48 & 91.55 & 95.39 & 100.00 \\
& {$[4.92,55.65]$} & {$[55.56,91.43]$} & {$[68.41,94.74]$} & {$[83.52,97.35]$} & {$[-]$} \\
$i_{t}^{s}$ & 8.95 & 66.99 & 76.00 & 84.60 & 100.00 \\
& {$[0.25,46.23]$} & {$[35.49,87.22]$} & {$[50.41,91.90]$} & {$[69.84,95.57]$} & {$[-]$} \\
$\hat{u}_{t}$ & 6.47 & 30.01 & 28.76 & 26.82 & 26.79 \\
& {$[0.03,26.07]$} & {$[1.57,62.07]$} & {$[2.02,62.10]$} & {$[2.30,61.03]$} & {$[2.33,60.90]$} \\
$\gamma_{t}$ & 8.71 & 40.76 & 51.56 & 63.31 & 100.00 \\
& {$[0.07,29.84]$} & {$[8.96,63.69]$} & {$[17.94,73.58]$} & {$[37.09,84.38]$} & {$[-]$} \\
$\hat{w}_{t}$ & 20.26 & 21.30 & 25.05 & 24.09 & 24.05 \\
& {$[0.22,39.44]$} & {$[2.25,38.29]$} & {$[2.70,45.37]$} & {$[3.08,45.60]$} & {$[3.17,45.87]$} \\
\hline & & & & & \\
\hline
\end{tabular}

Notes : les chiffres entre crochets sont les intervalles de confiance obtenus à l'aide d'une technique de bootstrap. Un - indique que l'intervalle de confiance est dégénéré.

fin, nous suivons GaLí et alii. [2003, 2007] et nous définissons la mesure d'inefficience $\left(\right.$ gap $\left._{t}\right)$ comme

$$
g a p_{t}=m r s_{t}-m p n_{t}
$$

où $m r s_{t}$ et $m p n_{t}$ sont respectivement le logarithme du taux marginal de substitution entre consommation et loisir et le logarithme de la productivité marginale du travail. Comme l'ont montré GALÍ et alii. [2007], la mesure gapt permet de quantifier simplement les coins sur les marchés des biens et du travail. En effet, la mesure d'inefficience peut être définie comme la somme de deux marges,

$$
\operatorname{gap}_{t}=-\left(\mu_{t}^{p}+\mu_{t}^{w}\right)
$$

où $\mu_{t}^{p}$ et $\mu_{t}^{w}$ représentent respectivement les logarithmes de la marge sur les prix et de la marge sur les salaires. La marge moyenne sur les prix dans l'économie est donnée par la différence entre la productivité marginale du travail et le salaire réel

$$
\mu_{t}^{p}=m p n_{t}-w_{t}
$$


tandis que la marge moyenne sur les salaires est donnée par la différence entre le salaire réel et le taux marginal de substitution entre loisir et consommation

$$
\mu_{t}^{w}=w_{t}-m r s_{t}
$$

où $w_{t}$ est le salaire réel. En faisant les hypothèses que les entreprises sont preneuses de prix sur le marché du travail et en l'absence de coût d'ajustement sur le facteur travail, la marge sur le prix $\mu_{t}^{p}$ est égale à la différence entre le prix des biens et le coût marginal nominal du travail. De façon symétrique, la marge sur les salaires est définie comme la différence entre le salaire réel et la désutilité du travail, les deux étant exprimées en unité de consommation. La marge sur les salaires peut inclure de nombreux coins, tels que les taxes sur le revenus du travail ou bien des salaires d'efficience (voir CHARI et alii., 2007). Cette décomposition permet d'une part d'évaluer comment la mesure globale d'inefficience a évolué suite à un choc de désinflation, mais aussi de mesurer le rôle respectif des marges sur les marchés des biens et du travail.

A ce stade, il est nécessaire de faire des hypothèses plus spécifiques sur les préférences et la technologie. Concernant les préférences, nous supposons que la fonction d'utilité consommation-loisir est de la forme fonctionnelle suivante

$$
u\left(C_{t}, N_{t}\right)=\log \left(C_{t}\right)-\bar{\Xi}_{t} \frac{N_{t}^{1+\phi}}{1+\phi}
$$

de sorte que le taux marginal de substitution est donné par

$$
m r s_{t}=c_{t}+\phi n_{t}+\bar{\xi}_{t}
$$

où $c_{t}$ représente le logarithme de la consommation par tête et $n_{t}$ le logarithme des heures par tête. Cette dernière est définie comme le produit des heures moyennes travaillées d'un individu et du nombre de personnes employées, le tout rapporté à la population en âge de travailler. Le paramètre $\phi \geq 0$ est l'inverse de l'élasticité Frishienne de l'offre de travail. La variable $\bar{\xi}_{t} \equiv \log \left(\bar{\Xi}_{t}\right)$ représente les mouvements longs qui affectent l'offre de travail. Ces mouvements peuvent représenter des changements institutionnels ou démographiques qui sont supposés non pertinents à la fréquence du cycle.

A ce niveau, la spécification du taux marginal de substitution $m r s_{t}$ pose deux problèmes de mesure. Le premier point concerne la valeur retenue pour le paramètre $\phi$. Dans notre expérience de référence, nous fixons $\phi=2$, une valeur proche de celle estimée par Smets et Wouters [2003] pour la zone euro ${ }^{10}$. Le second point concerne les propriétés dynamiques de $\bar{\xi}_{t}$. Nous supposons ici que ces chocs sur l'offre de travail sont non corrélés avec les chocs de désinflation.

Concernant la technologie, nous faisons l'hypothèse d'une fonction de production avec une élasticité constante par rapport au facteur travail. Il s'ensuit que, à un terme constant près, la productivité marginale du travail est donnée par

$$
m p n_{t}=y_{t}-n_{t},
$$


FIGURE 7 - Marges sur les prix et salaires et mesure d'inefficience
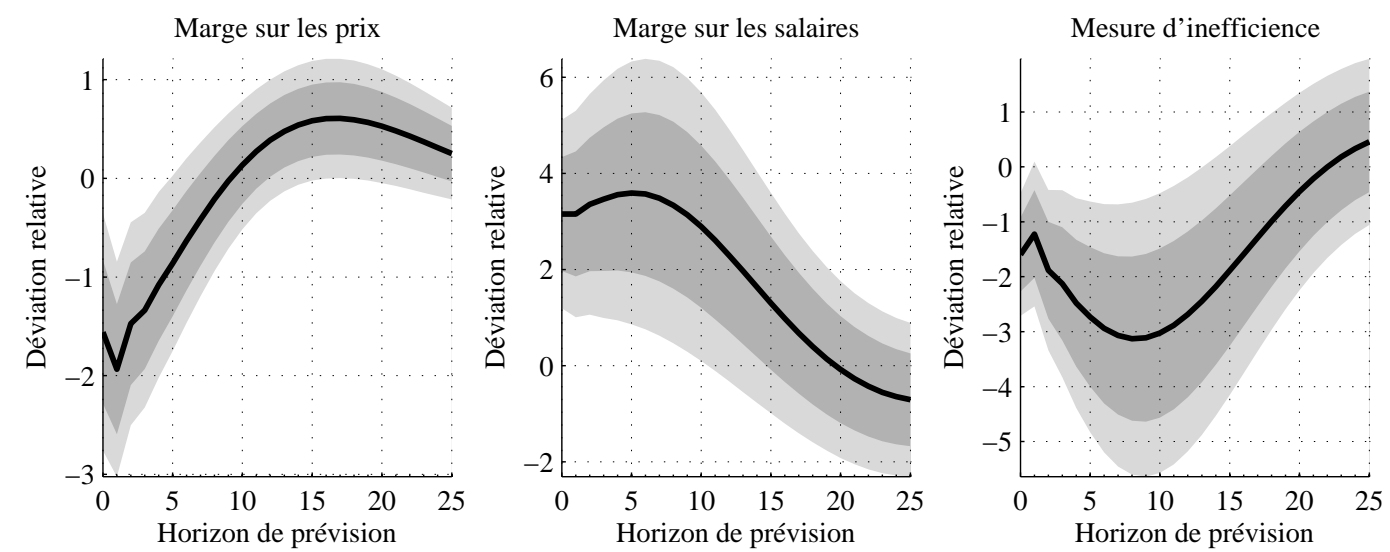

Notes : les aires grisées correspondent à l'intervalle de confiance à $90 \%$ (clair) et à $68 \%$ (foncé), obtenus à l'aide d'une technique de Bootstrap. Afin de faciliter l'interprétation, la taille du choc de désinflation est normalisée de sorte que la réponse asymptotique de l'inflation soit égale à $-1 \%$. Les calculs sont effectués sous l'hypothèse $\phi=2$.

Tableau 4. Décomposition de la variances des mesures d'inefficience

\begin{tabular}{cccccc}
\hline \hline & \multicolumn{5}{c}{ Horizon de prévision } \\
\cline { 2 - 6 } & 0 & 12 & 20 & 40 & $\infty$ \\
\hline$\hat{\mu}_{t}^{p}$ & 20.68 & 25.38 & 25.81 & 25.64 & 25.64 \\
& {$[0.25,43.69]$} & {$[2.74,48.81]$} & {$[2.95,48.75]$} & {$[2.99,49.39]$} & {$[2.99,49.49]$} \\
$\hat{\mu}_{t}^{w}$ & 32.00 & 30.68 & 28.95 & 28.14 & 28.14 \\
& {$[0.80,55.07]$} & {$[2.55,62.96]$} & {$[2.77,62.52]$} & {$[3.38,61.64]$} & {$[3.45,61.67]$} \\
$\widehat{\operatorname{gap}}_{t}$ & 16.79 & 27.95 & 27.21 & 26.21 & 26.19 \\
& {$[0.46,34.26]$} & {$[2.20,59.96]$} & {$[3.01,61.36]$} & {$[3.32,59.91]$} & {$[3.33,60.25]$} \\
\hline
\end{tabular}

Notes : les chiffres entre crochets sont les intervalles de confiance obtenus à l'aide d'une technique de bootstrap. Les calculs sont effectués sous l'hypothèse $\phi=2$.

où $y_{t}$ représente le logarithme du produit par tête.

A partir des définitions de $m p n_{t}$ et de $m r s_{t}$ et à l'aide des hypothèses ci-dessus, nous pouvons définir les composantes cycliques des mesures d'inefficience $\mu_{t}^{p}, \mu_{t}^{w}$ et gap , notées $\hat{\mu}_{t}^{p}$, $\hat{\mu}_{t}^{w}$ et $\widehat{g a p}_{t}$, respectivement. Ces composantes cycliques sont obtenues en substituant $\hat{w}_{t}, \hat{y}_{t}, \hat{c}_{t}$ et $\hat{n}_{t}$ au salaire réel, au produit, à la consommation et au heures travaillées dans les expressions précédentes. Comme le filtre à bande passante est linéaire, la composante cyclique de la somme est la somme des composantes cycliques. Cependant, le vecteur $Z_{t}$ dans le modèle (2) comprend la composante cyclique du taux de chômage, et non pas celle des heures travaillées par tête. Toutefois, en supposant qu'à court terme la participation sur le marché du travail et les heures de travail par individu ne changent pas trop après le choc de désinflation, la composante cyclique du taux de chômage satisfait $\hat{u}_{t} \simeq-\hat{n}_{t}$,

10. Nous étudions la sensibilité de nos résultats à la valeur de $\phi$. 
c'est à dire que sa déviation à la référence de long terme est approximativement égale (au signe moins près) à celle des heures travaillées. A l'aide du modèle VARS, nous sommes alors en mesure d'identifier les réponses de $\hat{\mu}_{t}^{p}, \hat{\mu}_{t}^{w}$ et $\widehat{g a p}_{t}$ au choc de désinflation que nous obtenons à partir des réponses des composantes cycliques du produit, de la consommation, du chômage et du salaire réel. Ces réponses sont reportées dans la figure 7 . Il convient de remarquer que, étant données nos définitions de l'inefficience sur les marchés des biens et du travail, une réponse négative de $\widehat{g a p}_{t}$ implique un accroissement global de l'inefficience (c'est à dire sur les deux marchés simultanément). De façon symétrique, une hausse de $\hat{\mu}_{t}^{p}$ (respectivement de $\hat{\mu}_{t}^{w}$ ) implique une baisse d'efficacité sur le marché des biens (respectivement sur le marché du travail).

La réponse négative et persistante de $\hat{\mu}_{t}^{p}$ indique que l'inefficience sur le marché des biens a diminué suite à la politique de désinflation. En d'autres termes, il semblerait que les marges des entreprises sur le prix des biens aient été sensiblement réduites. La marge sur les prix suit de façon très proche l'évolution du produit, révélant ainsi une forte pro-cyclicité (du moins suite à ce choc) dans la zone Euro. Concernant la marge sur les salaires, les résultats sont forts différents. Après un choc de désinflation, cette marge augmente instantanément et présente un profil en cloche pour les périodes suivantes. Ces mouvements contra-cycliques de la marge sur les salaires en presence de chocs de désinflation sont en accord avec ceux obtenus sur des données de la zone Euro par Galí et alii. [2003]. De plus la réponse positive de la marge sur les salaires excède (en valeur absolue) celle de la marge sur les prix. Il en découle que la mesure d'inefficience $\widehat{\operatorname{gap}}_{t}$ augmente de façon persistante après le choc de désinflation, suggérant ainsi que les distortions sur le marché du travail dans la zone Euro ont progressé suite à cette politique.

Nous calculons également la contribution du choc de désinflation à la variance des marges et de la mesure globale d'inefficience. Le tableau 4 reporte ces décompositions à différents horizons. Le choc de désinflation a un effet important sur ces mesures, ce qui est cohérent avec les effets réels de ce choc (voir le tableau 3). Il représente $25 \%$ de la variance de la marge sur les prix après trois ans et près de $31 \%$ de la marge sur les salaires. Dans le cas de la mesure globale, cette contribution est du même ordre quoique un peu plus faible (à peu près $28 \%$ ), puisque la réponse de la marge sur les prix vient pour partie contrebalancer celle de la marge sur les salaires. Pour des horizons plus importants, par exemple après dix ans, cette contribution est de l'ordre de $25 \%$. Bien qu'il ne soit pas une source dominante de fluctuations dans les mesures d'inefficience, le choc de désinflation représente une fraction non négligeable de leurs mouvements cycliques.

Nous évaluons la robustesse de ces résultats à différents choix du paramètres $\phi$. Les résultats avec différentes valeurs de $\phi$ comprises entre 0 et 5 sont reportés dans la figure 8 . Par construction, la marge sur les prix présente la même réponse que dans la figure 7. La réponse de la marge sur les salaires présente le même signe mais l'ampleur de la réponse diffère selon les valeurs de $\phi$. Quand $\phi$ prend des valeurs importantes, l'emploi répond peu de sorte que la marge sur les salaires augmente plus fortement. On obtient alors que la mesure d'inefficience croît plus fortement suite à un choc de désinflation. 
Figure 8 - Sensibilité à $\phi$
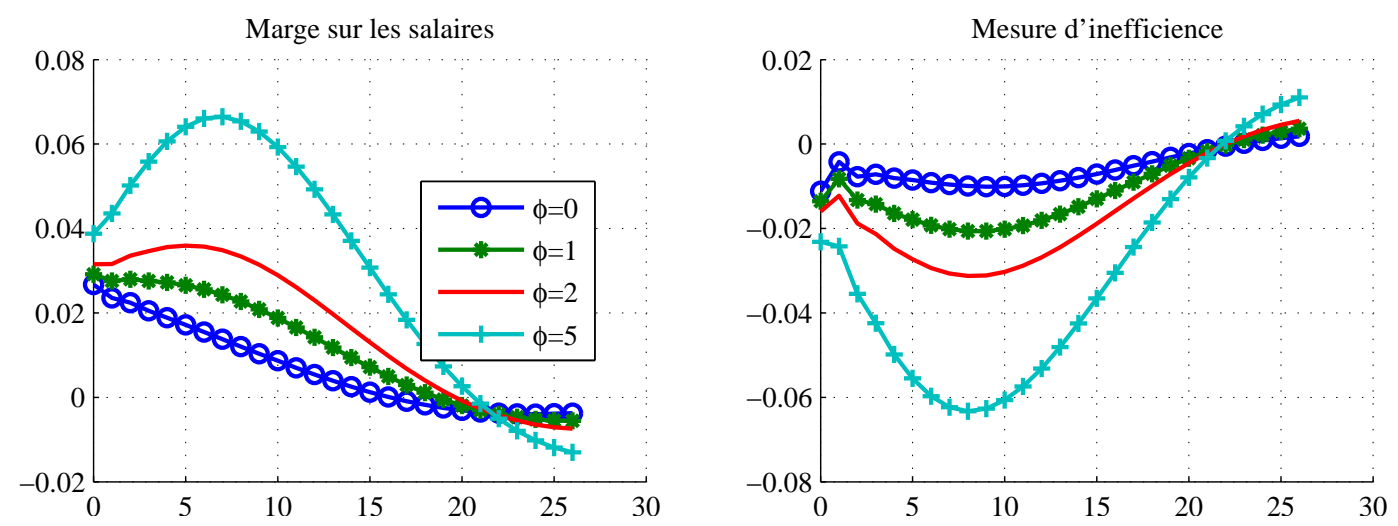

Notes : la figure ne reporte pas la réponse de la marge $\hat{\mu}_{t}^{p}$ sur le prix puisque celui-ci ne dépend pas de l'élasticité de l'offre de travail. La figure inclut la marge $\hat{\mu}_{t}^{w}$ sur le salaire ainsi que la mesure d'inefficience $\widehat{\operatorname{gap}}_{t}$ pour les valeurs $\phi=\{0,1,2,5\}$. Afin de simplifier la comparaison, nous ne reportons pas les intervalles de confiance.

\section{Robustesse des résultats}

Nous évaluons maintenant la robustesse de ces résultats à différentes modifications. Nous étudions successivement le rôle des variables étrangères (notamment le prix du pétrole et le taux de change réel), la définition des prix, les restrictions de long terme, la spécification du produit et du chômage et les origines non-monétaires des chocs de désinflation ${ }^{11}$.

\subsection{Prise en compte de variables étrangères}

Dans cet article, nous avons implicitement traité la zone euro comme une économie fermée, au sens où aucune variable extérieure à la zone n'a été prise en compte dans notre spécification de référence. Cette stratégie peut constituer un point de départ intéressant, étant donné la faible ouverture extérieure de la zone. Toutefois, il est important d'examiner la robustesse de nos résultats à la prise en compte de ces variables extérieures. Nous suivons PeERsman et Smets [2002] et incluons un vecteur de variables exogènes $X_{t}$ dans le modèle VAR. Comme ces auteurs, nous incluons dans $X_{t}$ le PIB américain en écart à une tendance linéaire qui nous sert d'approximation pour la demande mondiale, le taux de croissance des prix du pétrole et le taux de change réel $\$$ - euro $^{12}$. Le prix

11. Nous avons également évalué la sensibilité des résultats à une modification de la période d'estimation, par exemple en terminant l'échantillon au milieu des années quatre-vingt-dix. Les résultats obtenus sur cet échantillon plus court sont les mêmes pour les réponses, mais la précision des estimations s'avère fortement réduite. Ce résultat est assez standard dans la littérature VAR Structurel (voir Sims, 1998) qui montre que de tels modèles dynamiques peuvent produire des réponses relativement erratiques sur de petits échantillons. Nous avons aussi estimé un modèle de plus petite taille, comprenant la composante cyclique du produit, l'inflation, le taux de croissance de la monnaie et le taux de change réel. Les résultats sont similaires pour les variables nominales. En revanche, la réponse du produit est à la fois plus ample et moins persistante.

12. L'hypothèse de stationarité du PIB américain autour d'une tendance déterministe n'est pas rejetée par les tests de racine unitaire ADF et PP. Nos résultats sont préservés si nous substituons la composante cyclique du PIB 
Figure 9 - Robustesse à la prise en compte de variables étrangères exogènes
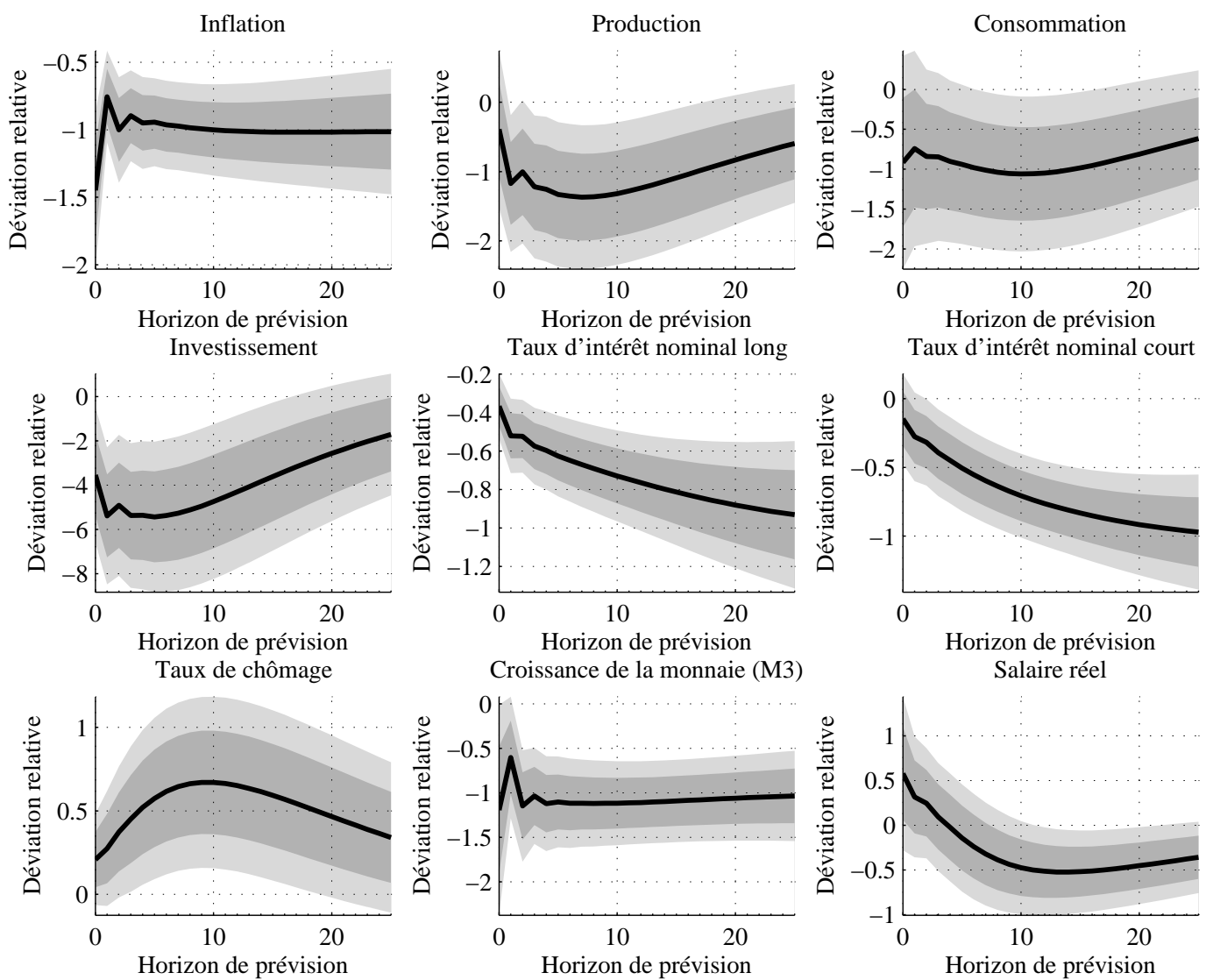

Notes : les aires grisées correspondent à l'intervalle de confiance à $90 \%$ (clair) et à $68 \%$ (foncé), obtenus à l'aide d'une technique de Bootstrap. Afin de faciliter l'interprétation, la taille du choc de désinflation est normalisée de sorte que la réponse asymptotique de l'inflation soit égale à $-1 \%$.

du pétrole est ici calculé comme le produit du West Texas Crude Oil Price et du taux de change nominal Euro-\$. En pratique, nous incluons la valeur contemporaine et un retard pour $X_{t}$. Les résultats sont reportés sur le graphique 9 .

Nous obtenons que la dynamique des variables nominales n'est pas affectée par la prise en compte de $X_{t}$. En revanche, nous trouvons que les effets sur les variables réelles sont près de deux fois moins amples mais beaucoup plus persistants. Au total, la valeur du ratio de sacrifice, qui se monte à 4.95 à cinq ans dans l'intervalle $[0.3636,8.2315]$, est pratiquement inchangée par rapport à notre spécification de référence. De ce point de vue, il est intéressant de remarquer que les effets dynamiques du choc de désinflation sur l'inflation ne doivent pas être confondus avec les effets du contre-choc pétrolier de la deuxième moitié des années $1980^{13}$.

américain obtenue par application du filtre de Christiano et Fitzgerald [2003].

13. Nous avons aussi inspecté les liens de causalité entre le choc de désinflation estimé dans notre spécification de référence du modèle (2) et le taux de croissance du prix du pétrole. Le test de causalité au sens de Granger est mené en régressant le choc de désinflation estimé sur une constante et les valeurs retardées du taux de croissance du prix de pétrole (de un à quatre retards). La statistique de Fisher donne un seuil du test de $73.75 \%$ tandis que celui de la statistique de Wald est égal à $71.69 \%$. Ces tests conduisent donc à rejeter cette causalité. Ces résultats confirment 
FiguRE 10 - Robustesse à la définition de l'inflation
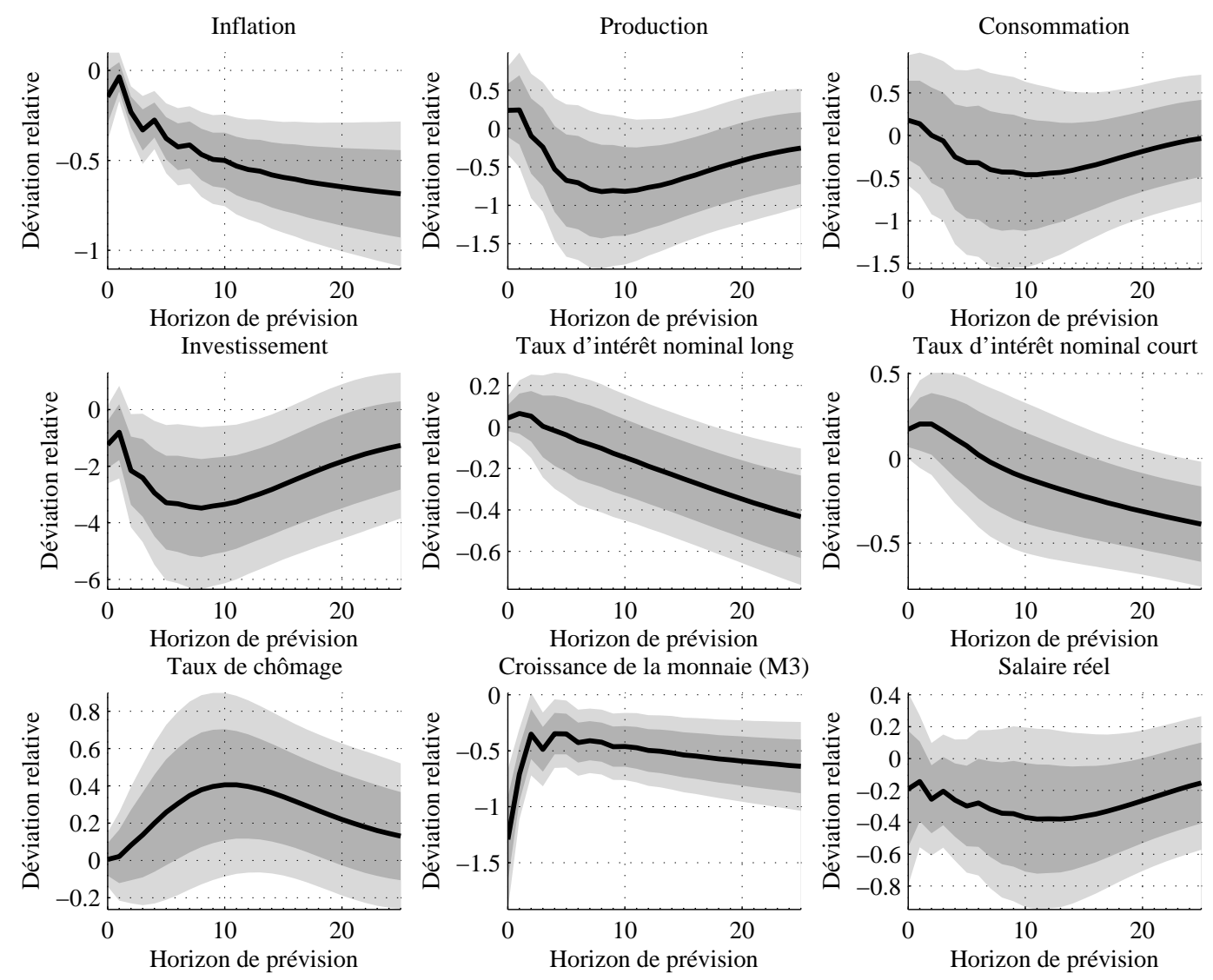

Notes : les aires grisées correspondent à l'intervalle de confiance à $90 \%$ (clair) et à $68 \%$ (foncé), obtenus à l'aide d'une technique de Bootstrap. Afin de faciliter l'interprétation, la taille du choc de désinflation est normalisée de sorte que la réponse asymptotique de l'inflation soit égale à $-1 \%$.

\subsection{Spécification des prix}

Dans notre spécification de référence, le taux d'inflation est obtenu à partir de l'indice de prix du PIB, comme dans la grande majorité des modèles VARS monétaires. Cependant, nous cherchons à évaluer si les réponses estimées sont sensibles à d'autres définitions de l'inflation dans la zone Euro. A cette fin, nous considérons l'indice de prix à la consommation (IPCH). En effet, cet indice de prix est connu comme étant celui suivi par la BCE (voir BCE, 2004) et comme le prix ayant servi de référence à la participation à la zone. Il apparaît donc légitime de l'utiliser afin d'identifier les effets d'un choc de désinflation.

Comme le montre la figure 10, cette nouvelle définition de l'inflation ne modifie que très faiblement nos précédentes conclusions. Néanmoins, quelques résultats intéressants apparaissent avec cette nouvelle définition. Tout d'abord, l'inflation présente un profil plus lisse d'ajustement vers sa nouvelle valeur de long terme. Ensuite, les effets de la politique de désinflation sont plus persistants. Par que le choc de désinflation identifié dans la spécification de référence n'est pas confondu avec les effets de l'importante baisse du prix du pétrole du milieu des années quatre-vingts. 
exemple, le produit atteint sa baisse maximale après dix trimestres au lieu de 8 dans notre spécification de référence. De même, la hausse maximale du taux de chômage apparaît après 10 trimestres au lieu de 8. Finalement, cette propriété empirique est partagée par presque toutes les variables. Enfin, dans la plupart des cas, nous obtenons des intervalles de confiance plus larges. Par exemple, la réponse du chômage n'est pas précisément estimée.

\subsection{Restrictions sur la relation de long terme entre variables nominales}

Nous évaluons maintenant les effets des restrictions cointégrantes entre les variables nominales. Contrairement à notre spécification de référence, nous n'imposons plus la restriction que les variables nominales incluses dans le vecteur $Z_{t}$ partagent la même tendance stochastique à long terme. Ainsi, le vecteur $Z_{t}$ est maintenant spécifié de la manière suivante

$$
Z_{t}=\left(\Delta \pi_{t}, \hat{y}_{t}, c_{t}-y_{t}, x_{t}-y_{t}, \hat{u}_{t}, \hat{w}_{t}, \Delta i_{t}^{s}, \Delta i_{t}^{l} \Delta \gamma_{t}\right)^{\prime}
$$

Nous maintenons la même spécification des composantes cycliques du produit, du chômage et du salaire réel. De plus, nous utilisons le même schéma d'identification du choc de désinflation. La figure 11 reporte les réponses dynamiques des différentes variables incluses dans $Z_{t}$. La comparaison avec la figure 4 montre que les restrictions cointégrantes sur les variables nominales n'ont que peu d'effet sur les réponses des variables réelles et nominales : (i) l'inflation, les taux d'intérêt de court et de long termes et le taux de croissance de la monnaie baissent suite au choc de désinflation, (ii) le produit, la consommation et l'investissement diminuent et (iii) le salaire réel et le chômage augmentent. La différence notable ne concerne pas le profil des réponses, mais leurs intervalles de confiance. Lorsque l'on n'impose pas ces relations de cointégration, la précision des réponses estimées diminue fortement. Cette absence de précision concerne non seulement les variables nominales mais aussi l'ensemble des variables réelles. Dans ce cas, le modèle VARS est tellement imprécis qu'il n'est plus possible d'obtenir des résultats clairs sur les effets agrégés des chocs de désinflation, à l'exception de la réponse de l'inflation.

\subsection{Spécification du chômage et du produit}

Dans notre spécification de référence, les mouvements cycliques du produit et du chômage sont obtenus par application du filtre de Christiano et FitzGerald [2003]. Etant donné le schéma d'identification, les chocs de désinflation ne peuvent donc pas avoir d'effets permanents sur le produit (et donc sur la consommation et l'investissement puisqu'ils apparaissent sous forme de ratios) et le taux de chômage. Suivant la littérature sur l'hystérèse, il est légitime d'évaluer si ces chocs peuvent avoir des effets permanents sur les variables réelles (voir BLANCHARD et SummERs, 1986 et Buiter et Miller, 1985). A cette fin, le produit et le chômage sont maintenant spécifiés en différence dans le vecteur $Z_{t}$

$$
Z_{t}=\left(\Delta \pi_{t}, \Delta y_{t}, c_{t}-y_{t}, x_{t}-y_{t}, \Delta u_{t}, \hat{w}_{t}, i_{t}^{l}-\pi_{t}, i_{t}^{l}-i_{t}^{s}, \gamma_{t}-\pi_{t}\right)^{\prime}
$$


FIGURE 11 - Robustesse aux restrictions de cointégration
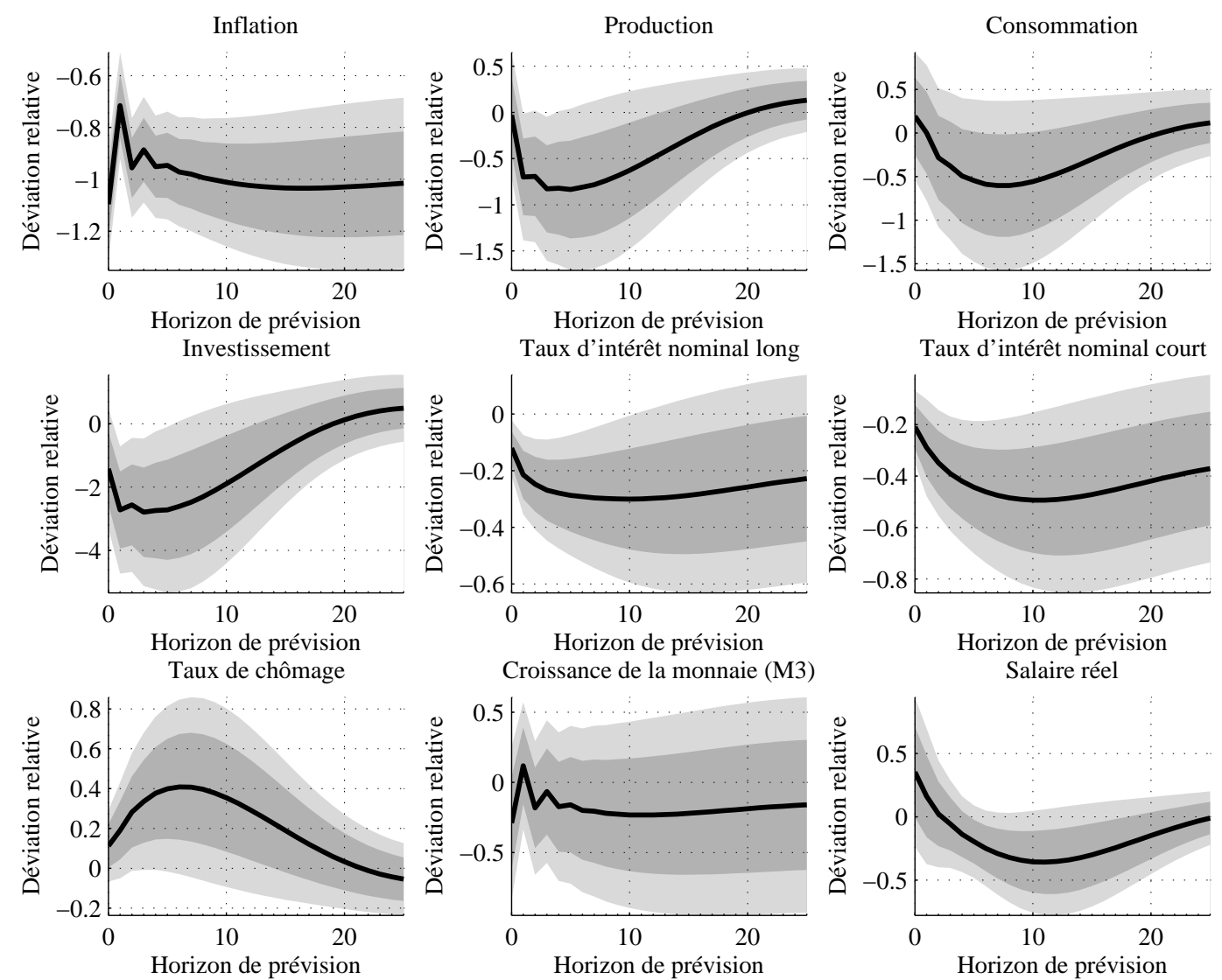

Notes : les aires grisées correspondent à l'intervalle de confiance à $90 \%$ (clair) et à $68 \%$ (foncé), obtenus à l'aide d'une technique de Bootstrap. Afin de faciliter l'interprétation, la taille du choc de désinflation est normalisée de sorte que la réponse asymptotique de l'inflation soit égale à $-1 \%$.

les autres variables étant spécifiées comme dans (1). Avec cette nouvelle spécification, la politique monétaire n'est plus contrainte à être neutre à long terme. Les résultats sont reportés dans le figure 12 .

Comme le montre la figure, toutes les variables, excepté le chômage, présentent les mêmes fonctions de réponse que dans la spécification de référence. En revanche, les intervalles de confiance sont plus larges. L'effet à long terme du choc de désinflation sur le produit est négatif, mais faible. Plus précisément, le choc de désinflation conduit à réduire asymptotiquement le produit par tête de $0.29 \%$ avec un intervalle de confiance de $[-1.9158 \%, 1.8420 \%]$. Si le produit contient une tendance stochastique, un choc de désinflation ne semble donc pas en expliquer une grande part. Ce résultat conforte donc le point de vue de la neutralité à long terme de ces chocs.

Concernant le taux de chômage, le profil des réponses est fortement modifié. Tout d'abord, la réponse maximale est maintenant deux fois plus forte que dans le cas de référence. Ensuite, la réponse à long terme ne présente pas de retour marqué et reste positive après 20 trimestres. Ce choc conduit à une hausse de 0.81 point du taux de chômage avec un intervalle de confiance de $[-0.8346 \%, 1.9145 \%]$. L'effet permanent du choc de désinflation sur le taux de chômage reste ainsi très imprécis. En 
FiguRE 12 - Robustesse à la spécification du produit et du chômage

Inflation
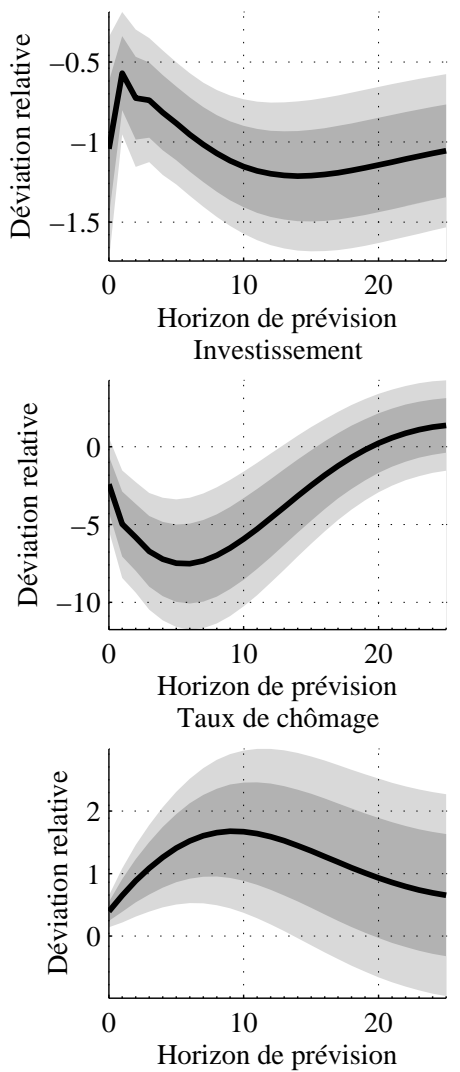

Production

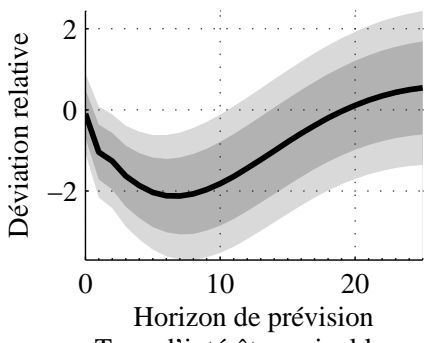

Taux d'intérêt nominal long
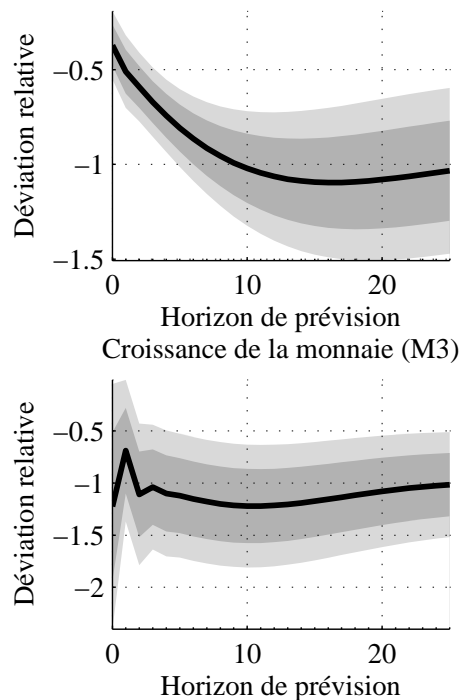

Consommation

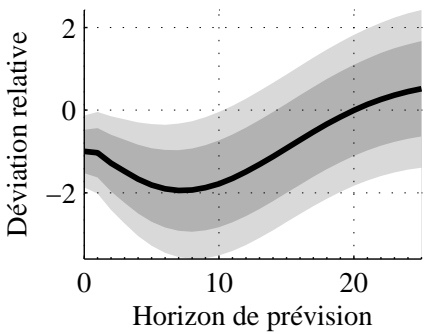

Taux d'intérêt nominal court
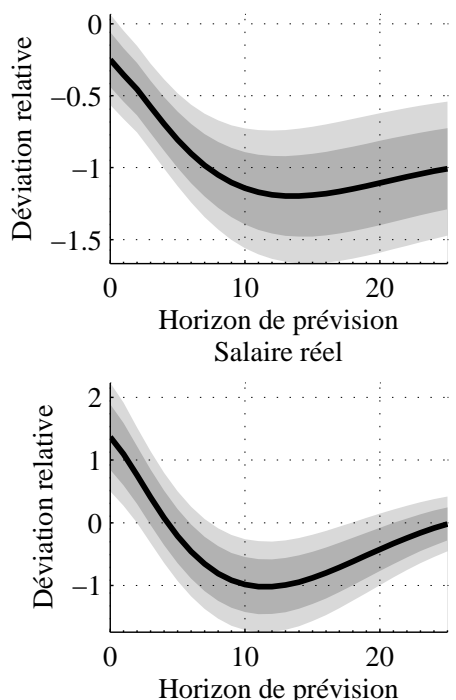

Notes : les aires grisées correspondent à l'intervalle de confiance à $90 \%$ (clair) et à $68 \%$ (foncé), obtenus à l'aide d'une technique de Bootstrap. Afin de faciliter l'interprétation, la taille du choc de désinflation est normalisée de sorte que la réponse asymptotique de l'inflation soit égale à $-1 \%$.

dépit de ces différences, il apparaît que la spécification de référence et celle autorisant un effet réel permanent délivrent un message assez proche concernant les effets récessionistes persistants des politiques de désinflation à court et à moyen termes.

\subsection{Relâchement de la doctrine monétariste}

Pour l'instant, nous avons imposé que seuls les chocs de désinflation peuvent avoir un effet permanent sur les variables nominales. Cette restriction est cohérente avec la doctrine monétariste (du moins à long terme) selon lequel que "l'inflation est toujours un phénomène monétaire" (FriEdman, 1968). Cette vision en écarte une autre, appelée "l'approche opportuniste" de la désinflation (voir Bomfim et Rudebusch, 2000 et Orphanides et Wilcox, 2002). Selon cette dernière, la banque centrale peut être tentée d'exploiter une expansion engendrée par un choc d'offre positif afin de mettre en œuvre une politique de désinflation. Si l'approche opportuniste s'avère la bonne, on devrait alors s'attendre à ce que différents chocs d'offre puissent avoir des effets à long terme sur l'inflation. Nous modifions alors notre schéma d'identification afin d'évaluer cette dernière approche. 
A cette fin, tout en maintenant le principe d'identification de long terme, nous modifions le vecteur $Z_{t}$ de la façon suivante

$$
Z_{t}=\left(\Delta u_{t}, \Delta y_{t}, \Delta \pi_{t}, c_{t}-y_{t}, x_{t}-y_{t}, \hat{w}_{t}, i_{t}^{l}-\pi_{t}, i_{t}^{l}-i_{t}^{s}, \gamma_{t}-\pi_{t}\right)^{\prime}
$$

En utilisant cette définition de $Z_{t}$ dans le modèle (2) et en utilisant la stratégie d'identification de BLANCHARD et QUAH [1989], nous imposons les restrictions à long terme suivantes : i) seul le choc de chômage, noté $\eta_{t}^{u}$, peut avoir un effet à long terme sur le taux de chômage; il peut aussi affecter asymptotiquement la production et l'inflation, ii) le choc de production, noté $\eta_{t}^{y}$, peut affecter de façon permanente le produit et l'inflation et iii) le choc de désinflation $\eta_{t}^{\pi}$ peut expliquer une partie de la dynamique de l'inflation à long terme, mais il n'a aucun effet à long terme sur le produit et le chômage. Etant donnée la spécification de $Z_{t}$, le choc de désinflation est contraint à nouveau à n'avoir aucun effet réel à long terme. Le choc de chômage peut s'interpréter comme un choc sur les coins du marché du travail qui modifie de façon permanente le facteur travail. On peut penser par exemple à des chocs permanents sur la taxation des revenus du travail ou bien encore un choc de préférence permanent sur l'offre de travail (voir Chang et alii., 2007, Dupaigne et alii., 2007). Le choc de production pourrait s'interpréter, suivant BLANCHARD et QUAH [1989], comme un choc technologique permanent. Cependant, comme l'a noté Galí [1999], des chocs modifiant de façon permanente le facteur travail (comme notre choc de chômage) peuvent affecter le produit à long terme. Notre choc de produit est donc un possible mélange de ces deux types de chocs. Le choc de désinflation reçoit quant à lui une interprétation identique à celle de notre spécification de référence, n'était qu'il n'explique plus à lui seul le niveau de long terme de l'inflation.

Les réponses au choc de désinflation sous cette représentation alternative sont reportées dans la figure 13. Nous obtenons un profil très similaire pour toutes les variables. La seule différence concerne l'amplitude et la persistance de l'effet récesionniste de la désinflation, qui apparaissent plus faibles mais se résorbent plus lentement. Ce résultat se reflète dans le ratio de sacrifice, qui est maintenant égal à $6.07 \%$ après cinq ans. Il faut noter que celui-ci n'est pas précisément estimé, faisant écho aux intervalles de confiance plus larges dans cette configuration.

Cet exercice permet également d'évaluer si les chocs "non-monétaires" contribuent à la dynamique de l'inflation à long terme. Les effets quantitatifs de ces chocs peuvent être directement obtenus à partir de la troisème ligne de la matrice de passage $S$. Les deux premières colonnes de cette ligne nous donnent les effets permanents sur l'inflation de $\eta_{t}^{u}$ et $\eta_{t}^{y}$, tandis que la troisième permet de mesurer la contribution du choc autonome de désinflation. En notant $I R F_{\infty}(\pi)$ la réponse globale de l'inflation à long terme, on peut décomposer cette dernière comme suit

$$
I R F_{\infty}(\pi)=\underset{(0.0012)}{-0.0002 \eta_{t}^{u}}-\underset{(0.0004)}{0.0005} \eta_{t}^{y}+\underset{(0.0004)}{0.0040} \eta_{t}^{\pi} .
$$

Dans cette dernière équation les chiffres entre parenthèses représentent les écart-types des effets à long terme des différents chocs. Afin d'interpréter plus structurellement cette équation, il faut d'abord s'assurer du statut des autres chocs. Par exemple, le choc $\eta_{t}^{y}$ a un effet positif sur le produit 
FigURE 13 - Robustesse au relâchement de la doctrine monétariste

Taux de chômage
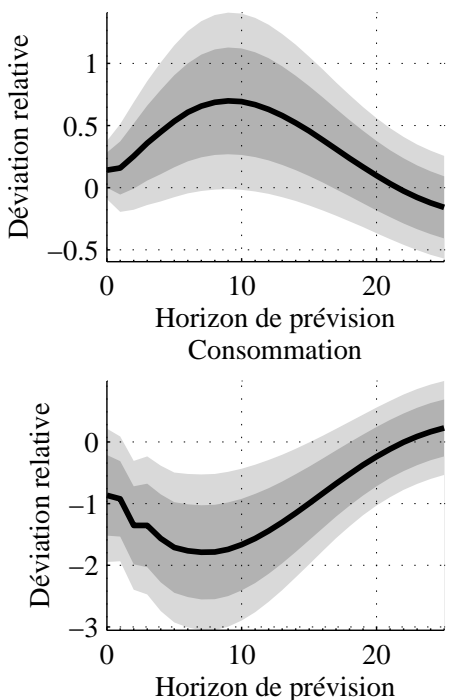

Taux d'intérêt nominal court

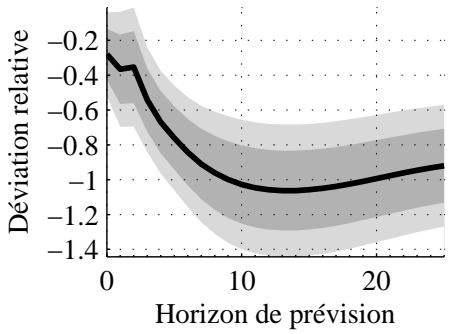

Production

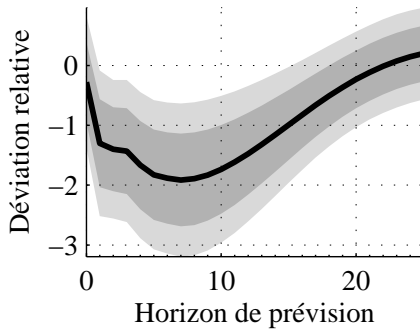

Investissement

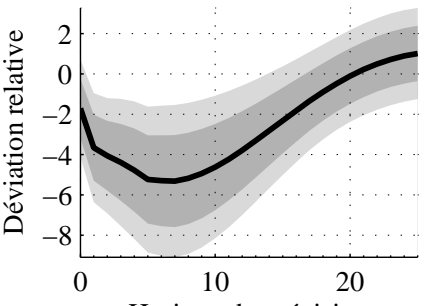

Horizon de prévision Croissance de la monnaie (M3)

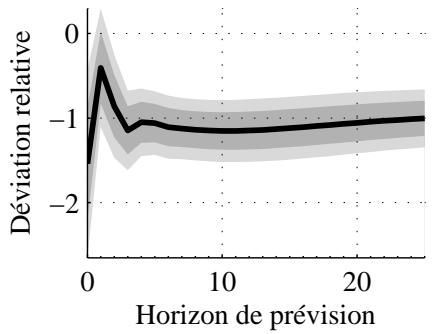

Inflation

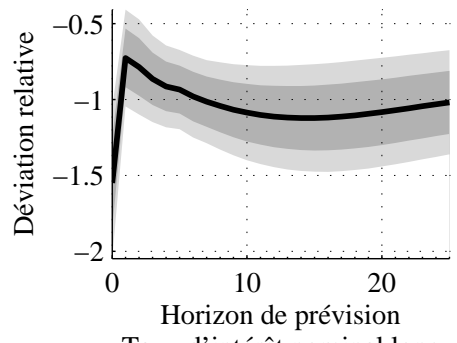

Taux d'intérêt nominal long

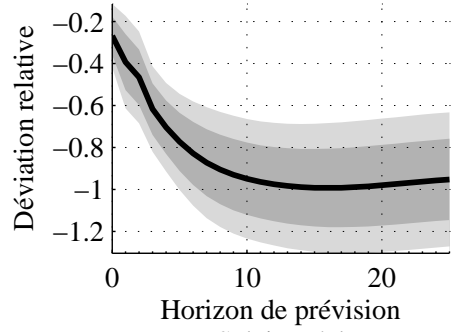

Salaire réel

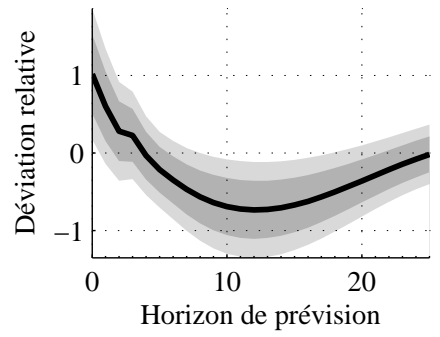

Notes : les aires grisées correspondent à l'intervalle de confiance à $90 \%$ (clair) et à $68 \%$ (foncé), obtenus à l'aide d'une technique de Bootstrap. Afin de faciliter l'interprétation, la taille du choc de désinflation est normalisée de sorte que la réponse asymptotique de l'inflation soit égale à $-1 \%$.

et un effet négatif sur l'inflation. Il est donc possible de le labéliser comme un choc d'offre. Le choc $\eta_{t}^{u}$ devrait quant à lui recevoir le statut de choc de demande puisqu'il fait baisser le produit et l'inflation. Cependant, en présence d'une approche opportuniste de la désinflation, la distinction choc d'offre-choc de demande devient délicate puisque, ici, la cible d'inflation et le niveau de la production covarient positivement.

Notre estimation montre que l'effet de $\eta_{t}^{y}$ est négatif, ce qui signifie, dans le cas d'une politique de désinflation, que la politique monétaire a cherché à tirer partie de ces chocs pour réduire l'inflation. Cet effet est ainsi en accord avec l'approche opportuniste ${ }^{14}$. Cependant, ce résultat doit être utilisé avec prudence car les écart-types des coefficients suggèrent que ces mécanismes ne sont pas significatifs. Nous obtenons une conclusion similaire en ce qui concerne $\eta_{t}^{u}$. Ces résultats sont d'ailleurs confirmés par la contribution asymptotique des deux chocs $\eta_{t}^{u}$ et $\eta_{t}^{y}$ à la dynamique de l'inflation. Ils représentent $16.61 \%$ de la variance de l'inflation avec un intervalle de confiance à $95 \%$ égal à $[0.67 \%, 80.64 \%]$. Ainsi, même si nous autorisons des contributions non-monétaires de l'inflation,

14. IRELAND [2007] obtient des résultats assez similaires pour les Etats-Unis à l'aide d'un modèle DSGE estimé par maximum de vraisemblance. 
FiguRE 14 - Cible d'inflation et approche opportuniste

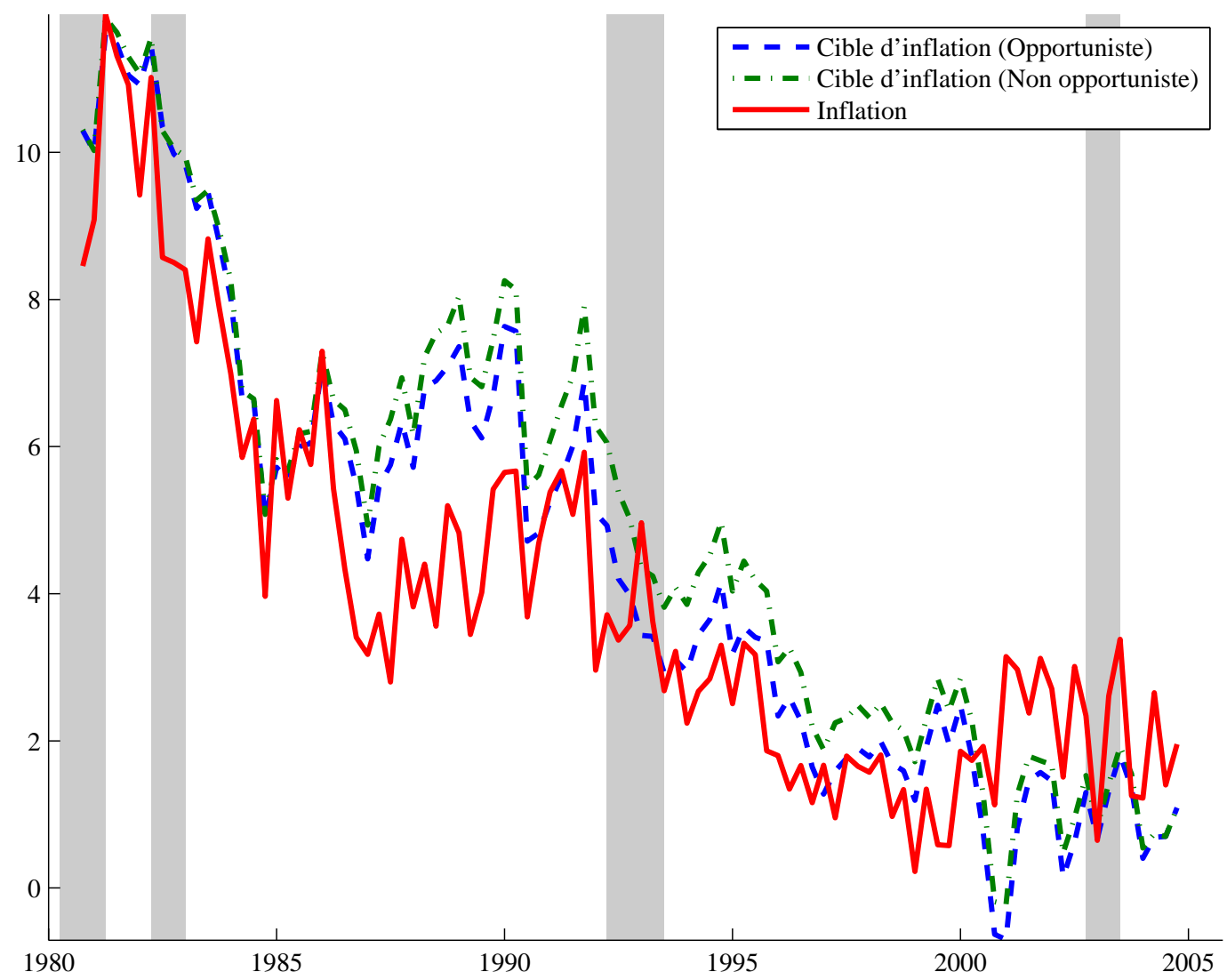

Notes : la cible d'inflation est identifiée à partir du modèle (4) en utilisant le schéma d'identification discuté dans le texte. La zone grisée correspond aux dates de récession identifiées à l'aide de l'algorithme de BryBoschan appliqué au logarithme du PIB par tête en niveau.

nous trouvons que la doctrine monétatiste est apparamment une dimension robuste de l'inflation dans la zone Euro.

Pour compléter cette analyse, nous proposons finalement de réinterpréter la cible implicite d'inflation dans le cadre de l'approche opportuniste. Pour ce faire, nous amendons l'équation (3) en y autorisant la présence des innovations $\eta_{t}^{u}$ et $\eta_{t}^{y}$. Formellement, l'équation de la cible implicite s'écrit

$$
\pi_{t}^{\star}=\pi_{t-1}^{\star}+\sigma_{\pi} \eta_{t}^{\pi}+\sigma_{u} \eta_{t}^{u}+\sigma_{y} \eta_{t}^{y}
$$

Dans cette équation, $\sigma_{u}$ est l'élément $3 \times 1$ de la matrice de passage $S, \sigma_{y}$ est l'élément $3 \times 2$ de $S$ et $\sigma_{\pi}$ est l'élément $3 \times 3$ de $S$. A des fins de comparaison, nous calculons une cible alternative sous l'hypothèse contre-factuelle que $\sigma_{u}=\sigma_{y}=0$. Les trajectoires ainsi obtenues sont reportées dans le graphique 14. Comme le laissaient entrevoir les effets de long terme des autres chocs structurels, nous n'obtenons pas de différence marquée entre les deux séries de cible d'inflation. Même s'il existe un effet opportuniste, les mouvements de basse fréquence de l'inflation semblent essentiellement d'origine monétaire. 


\section{Conclusion}

Dans cet article, nous utilisons la méthodologie VAR Structurel afin d'identifier les effets agrégés de chocs de désinflation dans la zone Euro. A l'aide de cette stratégie empirique, nous obtenons qu'un choc monétaire permanent de désinflation engendre des effets récessionnistes importants. Le produit par tête, la consommation et l'investissement baissent sensiblement tandis que le chômage présente une hausse persistante. Ces effets sont liés à une hausse persistante du taux d'intérêt réel. Le calcul des mesures d'inefficience sur les marchés du travail et des biens suggère toutefois que les marges sur les salaires, qui augmentent fortement après un choc de désinflation, ont joué un rôle non négligeable dans la propagation de ces chocs. Ces résulats apparaissent relativement robustes à d'autres définitions et spécifications des variables, ainsi qu'à d'autres représentations et identifications dans le modèle VAR Structurel. Les réponses éstimées de la zone, si elles sont prises plus ou moins pour argent comptant, peuvent représenter une nouvelle base de faits empiriques pour les modèles DSGE afin notamment d'expliciter plus clairement les mécanismes principaux (rigidités nominales et/ou réelles, degré de substitution des facteurs de production, conduite de la politique monétaire) responsables de ces effets observés. 


\section{Annexe A : Construction des données}

Les données brutes utilisées dans l'analyse et leur provenance sont détaillées dans le tableau A.1.

Tableau A.1. Données brutes

\begin{tabular}{llll}
\hline \hline Variable & Symbole & Mnémonique & Source \\
\hline PIB & $Y_{t}$ & YER & Base AWM, FAGAN et alii. [2005] \\
Consommation & $C_{t}$ & PCR & Base AWM, FAGAN et alii. [2005] \\
Investissement & $X_{t}$ & ITR & Base AWM, FAGAN et alii. [2005] \\
Chômage & $u_{t}$ & URX & Base AWM, FAGAN et alii. [2005] \\
Salaire & $W_{t}$ & WRN & Base AWM, FAGAN et alii. [2005] \\
Taux d'intérêt nominal court & $i_{t}^{s}$ & STN & Base AWM, FAGAN et alii. [2005] \\
Taux d'intérêt nominal long & $i_{t}^{l}$ & LTN & Base AWM, FAGAN et alii. [2005] \\
Déflateur du PIB & $P_{t}$ & YED & Base AWM, FAGAN et alii. [2005] \\
M3 & $M_{t}$ & - & Bulletin de la BCE \\
Population & $N_{t}$ & - & Perspectives économiques, OCDE \\
Prix du pétrole & - & - & Base Fred II \\
Taux de change Euro- $\$$ & - & EEN & Base AWM, FAGAN et alii. [2005] \\
PIB américain & - & & Base FREDII \\
Déflateur du PIB américain & - & & Base FREDII \\
\hline
\end{tabular}

Ces données brutes sont ensuite utilisées pour construire les variables utilisées dans l'analyse, selon la procédure décrite ci-dessous

$$
\begin{aligned}
y_{t} & =\log \left(Y_{t} / N_{t}\right) \\
c_{t} & =\log \left(C_{t} / N_{t}\right) \\
x_{t} & =\log \left(X_{t} / N_{t}\right) \\
w_{t} & =\log \left(W_{t}\right) \\
\pi_{t} & =\Delta \log \left(P_{t}\right) \\
\gamma_{t} & =\Delta \log \left(M_{t}\right)
\end{aligned}
$$

Les trajectoires historiques de $y_{t}, c_{t}-y_{t}, x_{t}-y_{t}, u_{t}, w_{t}, \pi_{t}, i_{t}^{s}, i_{t}^{l}$ et $\gamma_{t}$ sont reportées sur le graphique A.1. 
Figure A.1 - Données utilisées dans l'analyse
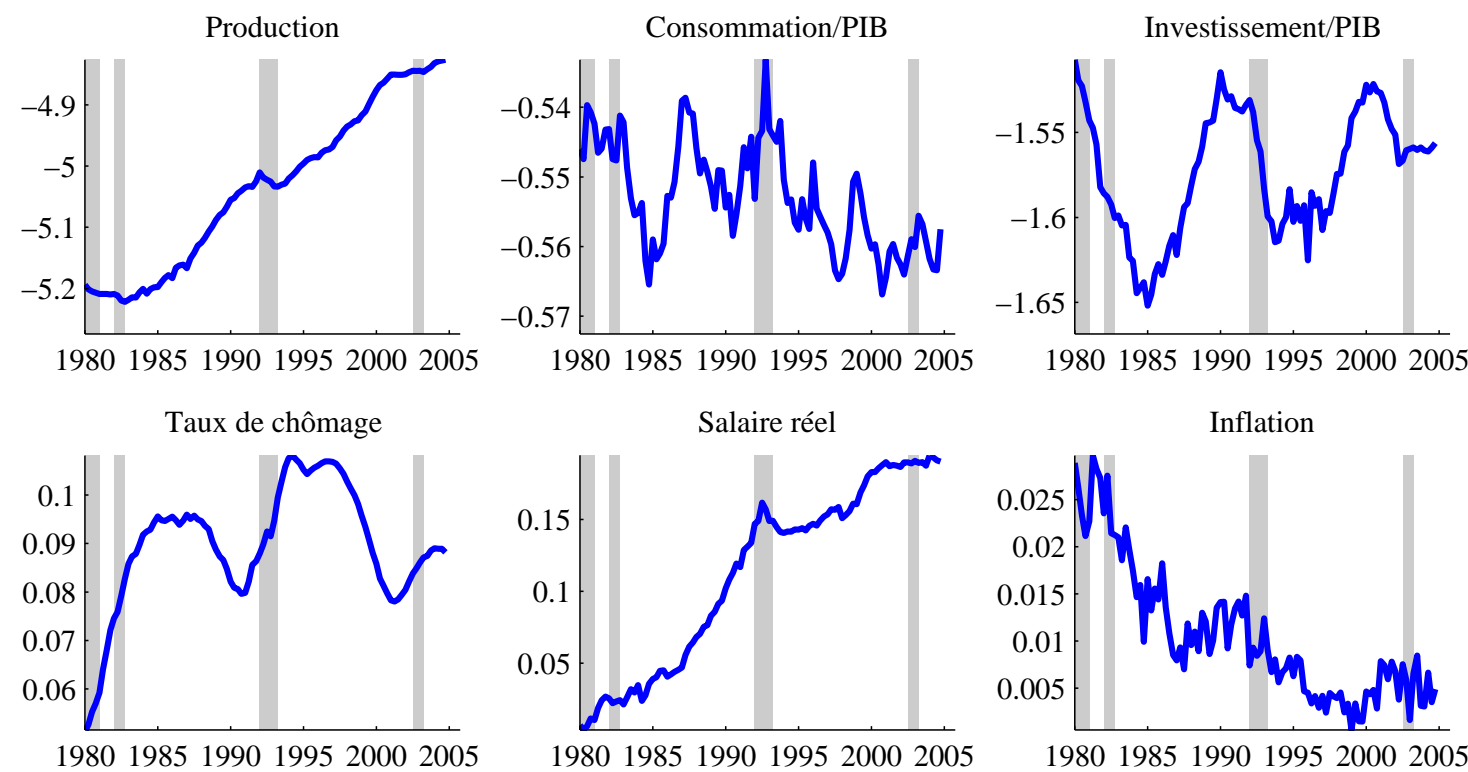

Taux d'intérêt nominal court
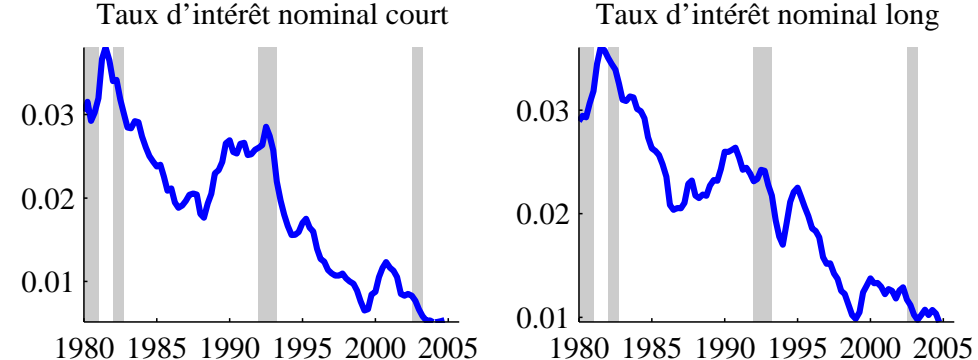

Croissance de la monnaie (M3)

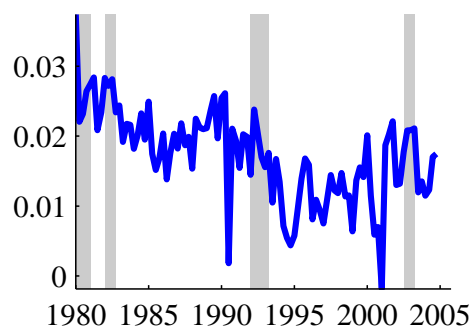

Notes : la zone grisée correspond aux dates de récession identifiées à l'aide de l'algorithme de Bry-Boschan appliqué au logarithme du PIB par tête en niveau. 
Figure A.2 - Trajectoires d'inflation des pays membres
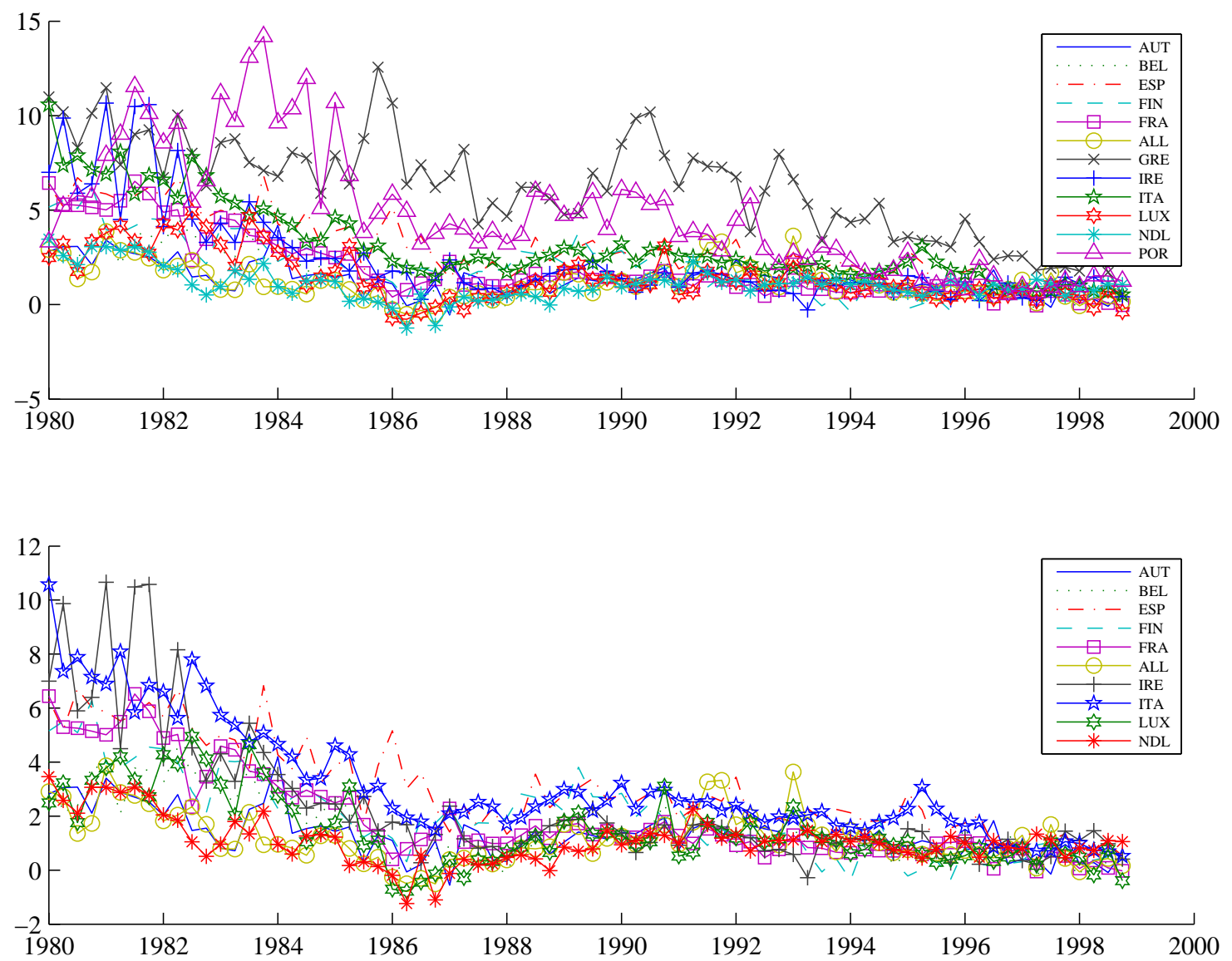

Notes : l'inflation est mesurée comme le taux de croissance de l'indice des prix à la consommation désaisonnalisé. Le panneau inférieur exclut la Grèce et le Portugal. 


\section{Références Bibliographiques}

Andersen P. et Wascher W. (1999) - «Sacrifice Ratios and the Conduct of Monetary Policy in Conditions of Low Inflation», Document de travail n`82, Banque des Règlements Internationaux.

Andres J. et Hernando I. (1999) - «Does Inflation Harm Economic Growth? : Evidence for the OECD Countries», in The Costs and Benefits of Achieving Price Stability, M. Feldstein (ed.). NBER and Michigan University Press. Boston.

Andres J., Hernando I. et López-Salido J. (2002) - «The Long-Run Effect of Permanent Disinflations», Document de travail n`9825, Banque d'Espagne.

Ball L. (1994) - «What Determines the Sacrifice Ratio?», in G. Mankiw (Ed.) Monetary policy, University of Chicago Press, 155-88.

Banque Centrale Européenne (2004) - The Monetary Policy of the ECB, Banque Centrale Européenne.

Blanchard O.J. (1997) - «The Medium Run», Brookings Papers on Economic Activity, 2, 89-158.

Blanchard O.J. (2003) - «Monetary Policy and Unemployement», remarques à la conférence Monetary Policy and the Labor Market, en l'honneur de James Tobin.

Blanchard O.J. et Summers L. (1986) - «Hysteresis and the European Unemployment Problem», NBER Macroeconomics Annual, 1, 15-78.

Blanchard O.J. et Muet P.A. (1993) - «Competitiveness through Disinflation : An Assessment of French Macroeconomic Policy since 1983», Economic Policy, 16, 12-56.

Blanchard O.J. et Quah D. (1989) - «The Dynamic Effects of Aggregate Demand and Supply Disturbances», American Economic Review, 79, 655-673.

Bomfim A.N. et Rudebusch G.D. (2000) - «Opportunistic and Deliberate Disinflation under Imperfect Credibility», Journal of Money, Credit, and Banking, 32, 701-21.

Buiter W. et Miller M. (1985) - «Costs and Benefits of an Anti-Inflationary Policy : Questions and Issues», in V. Argy et J. Neville (Eds.) Inflation and Unemployment :Theory, Experience and Policy Making, George Allen \& Unwin, Londres.

Bullard J. et Keating J. (1995) - «The Long-Run Relationship between Inflation and Output in Postwar Economies», Journal of Monetary Economics, 36, 477-496. 
Chang Y., Doh T. et Schorfheide, F. (2007) - «Non-Stationary Hours in a DSGE Model», Journal of Money, Credit, and Banking, 39, 1357-1373.

Christiano L.J., Eichenbaum M. et Evans C. (1999) - «Monetary Policy Shocks : What Have We Learned and to What End?», in M. Woodford et J. Taylor (Eds), Handbook of Macroeconomics, North-Holland, chapitre 3.

Christiano L.J., Fitzgerald T.J. (2003) - «The Band Pass Filter», International Economic Review, 44, 435-465.

Cling, P.P et Meunier F. (1986) - «La Désinflation en France : Le Point de Vue de l'Economètre», Revue Economique, 1093-1126.

Coenen G. et Vega J.L. (2001) - «The Demand for M3 in the Euro-Area», Journal of Applied Econometrics, 16, 727-748.

Cogley T. et Sargent T. (2007) - «Inflation-Gap Persistence in the U.S.», mimeo.

De Walque G., Smets, F. et Wouters R. (2006) - «Firm-Specific Production Factors in a DSGE Model with Taylor Price Setting», International Journal of Central Banking, 2, 107-154.

Dolado J., López-Salido J. et Vega J. (2000) - «Unemployement and Inflation Persistence in Spain : Are there Phillips Trade-Offs ?», Spanish Economic Review, 2, 267-291.

Dupaigne M., Fève P. et Matheron J. (2007) - «Avoiding Pitfalls in Using Structural VARs to Estimate Economic Models», Review of Economic Dynamics, 10, 238-255.

Fagan, G., Henry J. et Mestre R. (2005) - «An Area-Wide Model (AWM) for the Euro-Area», Economic Modelling, 22, 39-59.

Fitoussi J.P et Passet O. (2000) - «Réformes Structurelles et Politiques Macroéconomiques : Les Enseignements des Modèles de Pays», dans Réduction du Chômage : Les Réussites en Europe, Rapport n 23 du CAE, la Documentation Française.

Friedman M. (1968) - «Inflation : Causes and Consequences», in Dollars and Deficits : Living With America's Economic Problems, Prentice-Hall.

Galí J. (1999) - «Technology, Employment, and the Business Cycle : Do Technology Shocks Explain Aggregate Fluctuations ?», American Economic Review, 89, 249-271.

Galí J., Gertler M. et LóPez-Salido D. (2003) - «The Euro Area Inefficiency Gap», Document de travail n`0302, Banque d'Espagne. 
Galí J., Gertler M. et López-Salido D. (2007) - «Markups, Gaps and the Welfare Costs of Business Fluctuations», Review of Economics and Statistics, 89, 44-59.

Ireland P. (2007) - «Changes in the Federal Reserve's Inflation Target : Causes and Consequences», Journal of Money, Credit and Banking, 39, 1851-1882.

Kormendi R. et Meguire P. (1985) - «Macroeconomic Determinants of Growth : Cross Country Evidence», Journal of Monetary Economics, 16, 141-163.

McCandles G.T. et Weber W.E. (1995) - «Some Monetary Facts», Federal Reserve Bank of Minneapolis Quarterly Review, 19, 2-11.

Orphanides A. et Wilcox D.W. (2002) - «The Opportunistic Approach to Disinflation», International Finance, 5, 47-71.

Romer C. et Romer D. (1989) - «Does Monetary Policy Matter? A New Test in the Spirit of Friedman and Schwartz», NBER Macroeconomics Annual, 4, 121-170.

Romer C. et Romer D. (1994) - «Monetary Policy Matters», Journal of Monetary Economics, 34, 75-88.

Sims C.A. (1998) - «Comment on Glenn Rudebusch's "Do Measures of Monetary Policy in a VAR Make Sense?"», International Economic Review, 39, 933-941.

Smets F. et Wouters R. (2003) - «An Estimated Dynamic Stochastic General Equilibrium Model of the Euro-Area», Journal of the European Economic Association, 1, 1123-1175

Smets F. et Wouters R. (2005) - «Comparing Shocks and Frictions in US and Euro-Area Business Cycles : a Bayesian DSGE Approach», Journal of Applied Econometrics, 20, 161-183.

Staiger D., Stock J. et Watson M. (2002) - «Prices, Wages, and the US NAIRU in the 1990s», in Alan Krueger and Robert Solow (Eds.), The Roaring Nineties, Russel Sage Foundation, New York, 2002, 3-60 .

Stock J. et Watson M. (2007) - «Why has U.S. Inflation Become Harder to Forecast?», Journal of Money, Banking and Credit, 39, 3-34.

VlaAR P. (2004) - «Shocking the Eurozone», European Economic Review, 48, 109-131.

Watson M. (1994) - «Business-Cycle Durations and Postwar Stabilization of the U.S. Economy», American Economic Review, 84, 24-46. 